\title{
A New Compton-thick AGN in Our Cosmic Backyard: Unveiling the Buried Nucleus in NGC 1448 with NuSTAR
}

Annuar, A.; Alexander, D. M.; Gandhi, P.; Lansbury, G. B.; Asmus, K.-D.; Ballantyne, D. R.; Bauer, F. E.; Boggs, S. E.; Boorman, P. G.; Brandt, W. N.

Total number of authors:

25

Published in:

Astrophysical Journal

Link to article, DOI:

$10.3847 / 1538-4357 / 836 / 2 / 165$

Publication date:

2017

Document Version

Publisher's PDF, also known as Version of record

Link back to DTU Orbit

Citation (APA):

Annuar, A., Alexander, D. M., Gandhi, P., Lansbury, G. B., Asmus, K-D., Ballantyne, D. R., Bauer, F. E., Boggs, S. E., Boorman, P. G., Brandt, W. N., Brightman, M., Christensen, F. E., Craig, W. W., Farrah, D., Goulding, A. D., Hailey, C. J., Harrison, F. A., Koss, M. J., LaMassa, S. M., ... Zhang, W. (2017). A New Compton-thick AGN in Our Cosmic Backyard: Unveiling the Buried Nucleus in NGC 1448 with NuSTAR. Astrophysical Journal, 836(2), [165]. https://doi.org/10.3847/1538-4357/836/2/165

\section{General rights}

Copyright and moral rights for the publications made accessible in the public portal are retained by the authors and/or other copyright owners and it is a condition of accessing publications that users recognise and abide by the legal requirements associated with these rights.

- Users may download and print one copy of any publication from the public portal for the purpose of private study or research.

- You may not further distribute the material or use it for any profit-making activity or commercial gain

- You may freely distribute the URL identifying the publication in the public portal 


\title{
A New Compton-thick AGN in Our Cosmic Backyard: Unveiling the Buried Nucleus in NGC 1448 with NuSTAR
}

\author{
A. Annuar ${ }^{1}$, D. M. Alexander ${ }^{1}$, P. Gandhi ${ }^{2}$, G. B. Lansbury ${ }^{1}$, D. Asmus ${ }^{3}$, D. R. Ballantyne ${ }^{4}$, F. E. Bauer ${ }^{5,6,7}$, S. E. Boggs ${ }^{8}$, \\ P. G. Boorman ${ }^{2}$, W. N. Brandt ${ }^{9,10,11}$, M. Brightman ${ }^{12}$, F. E. Christensen ${ }^{13}$, W. W. Craig ${ }^{8,14}$, D. Farrah ${ }^{15}$, A. D. Goulding ${ }^{16}$, \\ C. J. Hailey ${ }^{17}$, F. A. Harrison ${ }^{12}$, M. J. Koss ${ }^{18}$, S. M. LaMassa ${ }^{19}$, S. S. Murray ${ }^{20,21,24}$, C. Ricci ${ }^{5,22}$, D. J. Rosario ${ }^{1}$, F. Stanley ${ }^{1}$, \\ D. Stern ${ }^{23}$, and W. Zhang ${ }^{19}$ \\ ${ }^{1}$ Centre for Extragalactic Astronomy, Department of Physics, Durham University, South Road, Durham, DH1 3LE, UK \\ ${ }^{2}$ Department of Physics \& Astronomy, Faculty of Physical Sciences and Engineering, University of Southampton, Southampton, SO17 1BJ, UK \\ ${ }^{3}$ European Southern Observatory, Alonso de Cordova, Vitacura, Casilla 19001, Santiago, Chile \\ ${ }^{4}$ Center for Relativistic Astrophysics, School of Physics, Georgia Institute of Technology, Atlanta, GA 30332, USA \\ ${ }^{5}$ Instituto de Astrofísica and Centro de Astroingeniería, Facultad de Física, Pontificia Universidad Católica de Chile, Casilla 306, Santiago 22, Chile \\ ${ }^{6}$ Millennium Institute of Astrophysics (MAS), Nuncio Monseñor Sótero Sanz 100, Providencia, Santiago, Chile \\ ${ }_{8}^{7}$ Space Science Institute, 4750 Walnut Street, Suite 205, Boulder, CO 80301, USA \\ ${ }^{8}$ Space Sciences Laboratory, University of California, Berkeley, CA 94720, USA \\ 9 Department of Astronomy and Astrophysics, The Pennsylvania State University, 525 Davey Lab, University Park, PA 16802, USA \\ ${ }^{10}$ Institute for Gravitation and the Cosmos, The Pennsylvania State University, University Park, PA 16802, USA \\ ${ }_{11}^{11}$ Department of Physics, The Pennsylvania State University, 525 Davey Lab, University Park, PA 16802, USA \\ ${ }^{12}$ Cahill Center for Astronomy and Astrophysics, California Institute of Technology, Pasadena, CA 91125, USA \\ ${ }^{13}$ DTU Space, National Space Institute, Technical University of Denmark, Elektrovej 327, DK-2800 Lyngby, Denmark \\ ${ }_{15}^{14}$ Lawrence Livermore National Laboratory, Livermore, CA 94550, USA \\ ${ }^{15}$ Department of Physics, Virginia Tech, Blacksburg, VA 24061, USA \\ ${ }_{17}^{16}$ Department of Astrophysical Sciences, Princeton University, Princeton, NJ 08544, USA \\ ${ }^{17}$ Columbia Astrophysics Laboratory, Columbia University, New York, NY 10027, USA \\ ${ }^{18}$ Institute for Astronomy, Department of Physics, ETH Zurich, Wolfgang-Pauli-Strasse 27, CH-8093 Zurich, Switzerland \\ ${ }^{19}$ NASA Goddard Space Flight Center, Greenbelt, Maryland 20771, USA \\ ${ }^{20}$ Harvard-Smithsonian Center for Astrophysics, 60 Garden Street, Cambridge, MA 02138, USA \\ ${ }^{21}$ Department of Physics and Astronomy, Johns Hopkins University, 3400 North Charles Street, Baltimore, MD 21218, USA \\ ${ }_{23}^{22}$ Kavli Institute for Astronomy and Astrophysics, Peking University, Beijing 100871, China \\ ${ }^{23}$ Jet Propulsion Laboratory, California Institute of Technology, Pasadena, CA 91109, USA \\ Received 2016 July 27; revised 2016 December 20; accepted 2016 December 27; published 2017 February 17
}

\begin{abstract}
NGC 1448 is one of the nearest luminous galaxies $\left(L_{8-1000 \mu \mathrm{m}}>10^{9} L_{\odot}\right)$ to ours $(z=0.00390)$, and yet the active galactic nucleus (AGN) it hosts was only recently discovered, in 2009. In this paper, we present an analysis of the nuclear source across three wavebands: mid-infrared (MIR) continuum, optical, and X-rays. We observed the source with the Nuclear Spectroscopic Telescope Array (NuSTAR), and combined these data with archival Chandra data to perform broadband X-ray spectral fitting $(\approx 0.5-40 \mathrm{keV})$ of the AGN for the first time. Our X-ray spectral analysis reveals that the AGN is buried under a Compton-thick (CT) column of obscuring gas along our line of sight, with a column density of $N_{\mathrm{H}}(\mathrm{los}) \gtrsim 2.5 \times 10^{24} \mathrm{~cm}^{-2}$. The best-fitting torus models measured an intrinsic $2-10 \mathrm{keV}$ luminosity of $L_{2-10 \text {,int }}=(3.5-7.6) \times 10^{40} \mathrm{erg} \mathrm{s}^{-1}$, making NGC 1448 one of the lowest luminosity CTAGNs known. In addition to the NUSTAR observation, we also performed optical spectroscopy for the nucleus in this edge-on galaxy using the European Southern Observatory New Technology Telescope. We reclassify the optical nuclear spectrum as a Seyfert on the basis of the Baldwin-Philips-Terlevich diagnostic diagrams, thus identifying the AGN at optical wavelengths for the first time. We also present high spatial resolution MIR observations of NGC 1448 with Gemini/T-ReCS, in which a compact nucleus is clearly detected. The absorption-corrected $2-10 \mathrm{keV}$ luminosity measured from our X-ray spectral analysis agrees with that predicted from the optical [O III] $\lambda 5007 \AA$ emission line and the MIR $12 \mu \mathrm{m}$ continuum, further supporting the CT nature of the AGN.
\end{abstract}

Key words: galaxies: active - galaxies: nuclei - techniques: spectroscopic - X-rays: galaxies - X-rays: individual (NGC 1448)

\section{Introduction}

At a distance of $11.5 \mathrm{Mpc}(z=0.00390),{ }^{25} \mathrm{NGC} 1448$ is one of the nearest bolometrically luminous galaxies $\left(L_{8-1000 \mu \mathrm{m}}>10^{9} L_{\odot}\right)$ to our own. Yet, the presence of an active galactic nucleus (AGN) at the center of the galaxy was

\footnotetext{
24 Deceased.

25 The quoted distance is the metric/proper distance calculated based upon the Mould et al. (2000) cosmic attractor model, using $H_{0}=75 \mathrm{~km} \mathrm{~s}^{-1} \mathrm{Mpc}^{-1}$ and adopting flat cosmology $\left(\Omega_{M}=0.3, \Omega_{\Lambda}=0.7, q_{0}=0.3\right)$ (Sanders et al. 2003).
}

only discovered less than a decade ago by Goulding \& Alexander (2009) through the detection of the high-ionization $[\mathrm{NeV}] \lambda 14.32 \mu \mathrm{m}$ emission line, as part of a sample of luminous galaxies observed by Spitzer. Based on the [Ne V]/ $f_{8-1000 \mu \mathrm{m}}$ and $[\mathrm{Ne} \mathrm{V}] /[\mathrm{Ne}$ II $] \lambda 12.82 \mu \mathrm{m}$ luminosity ratios, they found that the AGN contributes a significant fraction $(>25 \%)$ of the infrared (IR) emission. So far, the focus of the majority of studies of NGC 1448 has been on a number of supernovae occuring in the galaxy (e.g.; Wang et al. 2003; Sollerman et al. 2005; Monard et al. 2014). 
Based on its total $K$-band magnitude from the Two Micron All-Sky Survey (2MASS) Large Galaxy Atlas, $K_{\text {Tot }}=7.66$ (Jarrett et al. 2003), the total mass of NGC 1448 is $\log \left(M_{\mathrm{gal}} /\right.$ $\left.M_{\odot}\right)=10.3$ (using the stellar mass-to-light ratio versus $B-V$ relation of Bell et al. 2003.) The star formation rate (SFR) of the galaxy estimated from its far-IR luminosity measured by the Infrared Astronomical Satellite (IRAS), $\log L_{\mathrm{fir}}=9.70 L_{\odot}$ (Sanders et al. 2003), is SFR $\sim 1 M_{\odot} \mathrm{yr}^{-1}$ (Kennicutt 1998). This is consistent with the SFR expected at this redshift, given the mass of the galaxy (Davé 2008). The nucleus is classified as an H II region in the optical by Veron-Cetty \& Veron (1986) using the $\mathrm{H} \alpha$ to $[\mathrm{N} \mathrm{II}] \lambda 6583 \AA$ line ratio $(\mathrm{H} \alpha /[\mathrm{N} \mathrm{II}]>1.7)$, without any clear evidence for an AGN. The [O III] $\lambda 5007 \AA$ and $\mathrm{H} \beta$ emission lines were not detected in their observation, which could be attributed to obscuration by the highly inclined Scd host galaxy $\left(i \approx 86^{\circ}\right.$ relative to the plane of the sky). ${ }^{26}$ Based on the [O IV] $\lambda 25.89 \mu \mathrm{m}$ to [O III] $\lambda 5007$ A lower limit emission line ratio, Goulding \& Alexander (2009) found an extinction of $A_{V}>5 \mathrm{mag}$ within the galaxy, suggesting high obscuration toward the AGN.

In recent years, many studies have been conducted to find the most heavily obscured AGNs, particularly Compton-thick (CT) AGNs, in the local universe. CTAGNs are AGNs that are obscured along our line of sight by gas with a column density of $N_{\mathrm{H}} \geqslant 1 / \sigma_{T}=1.5 \times 10^{24} \mathrm{~cm}^{-2}$ (where $\sigma_{T}$ is the Thomson scattering cross-section). The obscuring gas is predominantly attributed to the AGN circumnuclear torus posited by the AGN unification model (Antonucci 1993; Urry \& Padovani 1995), but can also be contributed by larger-scale molecular clouds and dust lanes (e.g., Elvis 2012; Prieto et al. 2014). The high column of gas severely absorbs the direct X-ray emission from the $\mathrm{AGN}$, even at $E \gtrsim 10 \mathrm{keV}$ in cases where the absorption is extreme $\left(N_{\mathrm{H}}>10^{25} \mathrm{~cm}^{-2}\right.$; see Figure 1 of Gilli et al. 2007). This is what makes it very challenging to identify CTAGNs. Indeed, a hard X-ray study $(E>10 \mathrm{keV})$ by Ricci et al. (2016) using a large AGN sample from the Swift Burst Alert Telescope (BAT) 70 month survey catalog, found an observed CTAGN fraction of $\sim 8 \%$ at $z \simeq 0.055$. This is significantly lower than that expected from the synthesis of the cosmic X-ray background spectrum (10\%25\%; e.g., Gilli et al. 2007; Treister et al. 2009; Draper \& Ballantyne 2010; Akylas et al. 2012; Ueda et al. 2014), and what is predicted from multiwavelength studies of nearby AGN ( 30\%; e.g., Risaliti et al. 1999; Goulding et al. 2011). This suggests that we are still missing a significant number of CTAGNs, even in the local universe. Forming a complete census of their population is important in understanding the cosmic X-ray background spectrum and the growth of supermassive black holes.

In this paper, we present Nuclear Spectroscopic Telescope Array (NuSTAR) and Chandra observations of NGC 1448 in which the AGN is detected in X-rays for the first time. We find that our direct view toward the AGN is hindered by a CT column of obscuring gas. We also report the results of new high angular resolution mid-infrared (MIR) and optical observations of the source by Gemini/T-ReCS and New Technology Telescope (NTT)/EFOSC2, respectively, which also reveal the presence of a buried AGN. The paper is organized as follows. In Section 2, we describe details of the multiwavelength observations and data reduction procedures

\footnotetext{
${ }^{26}$ We obtained the host galaxy inclination from the HyperLeda website (http://leda.univ-lyon1.fr/).
}

for NGC 1448. We present the X-ray spectral modeling and results in Section 3, followed by the data analysis and results of the optical and MIR observations in Section 4. Finally, we discuss and summarize the overall results in Sections 5 and 6, respectively.

\section{Observations}

In this section, we describe the new NUSTAR (Sections 2.1) and archival Chandra (Sections 2.2) observations of NGC 1448. The NuSTAR data are essential for tracing the intrinsic emission from the buried AGN at high X-ray energies $(E \gtrsim 10 \mathrm{keV})$. The Chandra data were used to aid our X-ray spectral analysis of the AGN at lower energies $(E \lesssim 3 \mathrm{keV})$ where $N u S T A R$ is not sensitive, and to reliably account for the emission from contaminating off-nuclear X-ray sources in the NUSTAR spectrum. Combining both NUSTAR and Chandra data together allows us to analyze the X-ray spectrum of the AGN in NGC 1448 over a broadband range of energy for the first time. We also describe new optical data (spectroscopy and imaging) obtained with the European Southern Observatory (ESO) NTT (Section 2.3), and the new high angular resolution MIR observations with the Gemini-South telescope (Section 2.4), to provide a multiwavelength view of the AGN.

In addition to these data, we note that NGC 1448 has also been observed by Suzaku in X-rays for an exposure time of $\approx 53 \mathrm{ks}$ in 2009 (2009 February 17; PI D. M. Alexander; ObsID 703062010). However, it is only significantly detected in the $\mathrm{X}$-ray Imaging Spectrometer (XIS) instrument up to $\sim 10 \mathrm{keV}$. Analysis of the deepest Swift-BAT 104 month maps using custom detection techniques to look for faint sources (Koss et al. 2013) show no significant excess (signal-to-noise ratio, $\mathrm{S} / \mathrm{N}=-0.1)$ in the area near NGC 1448 . The source was only detected in 1 out of 5 Swift X-ray Telescope (XRT) observations with $\sim 12$ counts in $\sim 10 \mathrm{ks}$ exposure time (2009 November 28; ObsID 00031031001). We do not include these data in our X-ray analysis as they do not provide additional constraints beyond those already achieved with our NuSTAR and Chandra data.

\subsection{NUSTAR}

NuSTAR (Harrison et al. 2013), launched in 2012 June, is the first orbiting X-ray observatory which focus light at high energies $(E \gtrsim 10 \mathrm{keV})$. It consists of two co-aligned focal plane modules (FPMs), which are identical in design, and referred to as FPMA and FPMB. Each FPM covers the same $12^{\prime} \times 12^{\prime}$ portion of the sky, and comprises four cadmiumzinc-telluride detectors placed in a $2 \times 2$ array. NUSTAR operates between 3 and $79 \mathrm{keV}$, and provides $100 \times$ improvement in sensitivity as compared to previous hard X-ray orbiting observatories ( $E \gtrsim 10 \mathrm{keV}$; e.g., Swift-BAT and INTEGRAL). In addition, it has an angular resolution of $18^{\prime \prime}$ full-width at half-maximum (FWHM), with a half power diameter of 58", resulting in $10 \times$ improvement over previous observatories operating at $E \gtrsim 10 \mathrm{keV}$. These characteristics, particularly its high energy coverage and better sensitivity, have allowed us to perform accurate broadband X-ray spectral modelings of heavily obscured AGNs in the local universe (e.g., Baloković et al. 2014; Puccetti et al. 2014; Annuar et al. 2015; Bauer et al. 2015; Gandhi et al. 2016).

NGC 1448 was observed by NuSTAR in 2015 (2015 July 12; ObsID 60101101002) with an effective exposure time of 


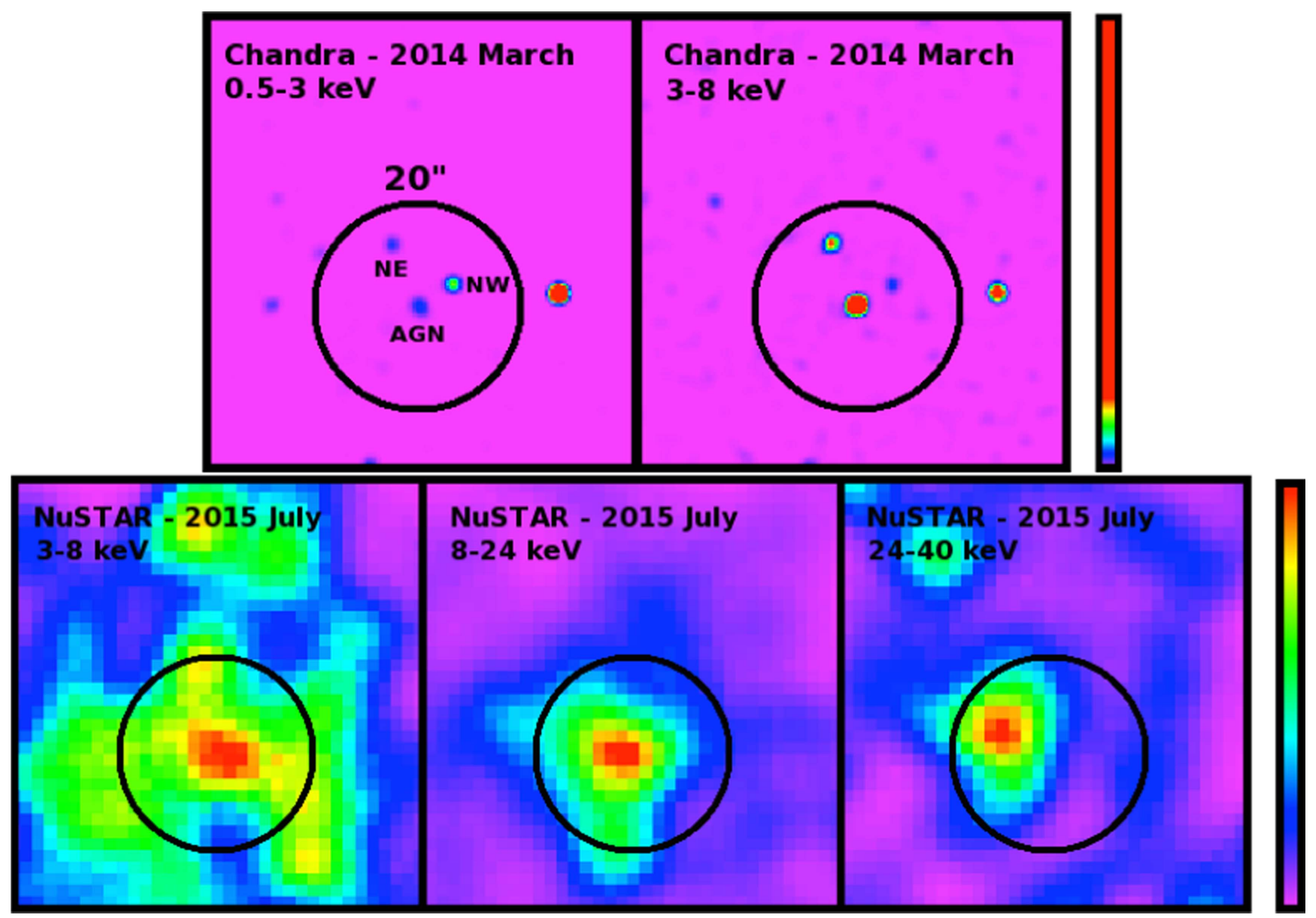

Figure 1. Chandra images of NGC 1448 in the $0.5-3 \mathrm{keV}$ and $3-8 \mathrm{keV}$ bands (top panel) and NuSTAR FPMA+B images in the 3-8 keV, 8-24 keV, and 24-40 keV bands (bottom panel). The color scales for Chandra and NuSTAR images are shown with magenta and red representing the lowest and highest counts in each image, respectively. The black circle marks a $20^{\prime \prime}$ radius region centered on the Chandra position of the AGN that was used to extract the X-ray spectra. Images are smoothed with a Gaussian function of radius 5 pixels, corresponding to 2 " 47 and 12". 3 for Chandra and NuSTAR, respectively. North is up and east is to the left in all images. The two off-nuclear X-ray sources detected within the extraction region in the Chandra image are at the north-east and north-west of the AGN, labeled as NE and NW, respectively.

58.9 ks (60.3 ks on-source time) for each FPM. The source was observed as part of our program to form the most complete census of the CTAGN population and the $N_{\mathrm{H}}$ distribution of AGNs in the local universe, using a volume-limited $(D<15 \mathrm{Mpc})$ AGN sample from Goulding \& Alexander (2009). ${ }^{27}$

We processed the NuSTAR data of NGC 1448 with the NUSTAR Data Analysis Software (NUSTARDAS) v1.4.1 within HEASOFT v6.15.1 with CALDB v20150316. The NUPIPELINE v0.4.3 script was used to produce calibrated and cleaned event files using standard filter flags. We extracted the spectra and response files using the NUPRODUCTS v0.2.5 task.

The AGN is detected in both of the NuSTAR FPMs. The combined images of the AGN from the two FPMs in the 3-8, $8-24$, and $24-40 \mathrm{keV}$ bands are shown in Figure 1. We extracted the NUSTAR spectrum of NGC 1448 from each FPM using a circular aperture region of $20^{\prime \prime}$ radius (corresponding to $\sim 30 \%$ NUSTAR encircled energy fraction, ECF) centered on the Chandra position of the AGN (see Section 2.2). The aperture size was chosen to minimize contamination from off-nuclear sources observed in the Chandra data. Background photons were collected from an

\footnotetext{
27 The results of the first source in the sample observed by NUSTAR as part of this program, NGC 5643, was reported in Annuar et al. (2015).
}

annulus region centered on the AGN with inner and outer radii of $40^{\prime \prime}$ and $70^{\prime \prime}$, respectively. The extracted spectrum from each FPM was then co-added using the ADDASCASPEC script to increase the overall $\mathrm{S} / \mathrm{N}$ of the data. We detected significant counts up to $\sim 40 \mathrm{keV}$ from this combined spectrum, and measured a net count rate of $2.79 \times 10^{-3}$ counts $\mathrm{s}^{-1}$ in the $3-40 \mathrm{keV}$ band.

We note that in the $24-40 \mathrm{keV}$ band image, where we expect the AGN to completely dominate, the peak emission appears to be offset from the center of the extraction region. The offset is not observed in the 3-8 and $8-24 \mathrm{keV}$ band images where NUSTAR is more sensitive. Performing our spectral fits up to only $24 \mathrm{keV}$ gave consistent results with that obtained by the $0.5-40 \mathrm{keV}$ spectral fits. We therefore attribute this offset due to NUSTAR statistical uncertainty due to lower $\mathrm{S} / \mathrm{N}$ at this higher energy band. We also note that centering the NUSTAR extraction region according to this offset or enlarging the extraction region to account for this apparent offset do not significantly affect our final results on the analysis of the AGN in NGC 1448.

\subsection{Chandra}

NGC 1448 was observed by Chandra in 2014 with an exposure time of $49.4 \mathrm{ks}$ ( $50.1 \mathrm{ks}$ on-source time) using the 


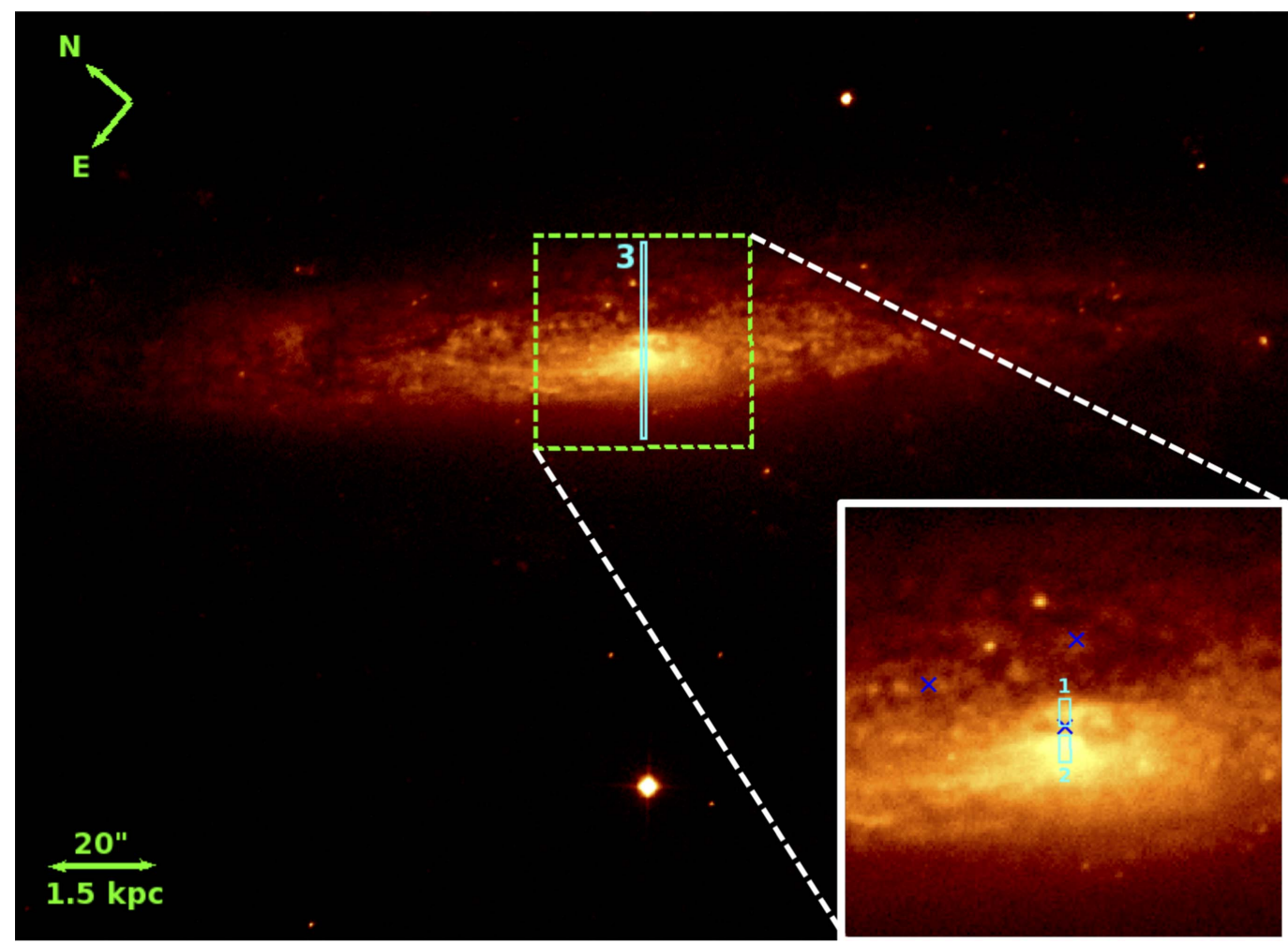

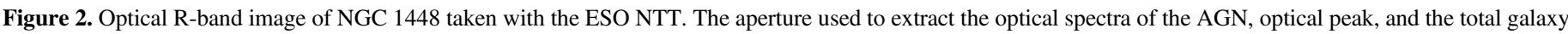

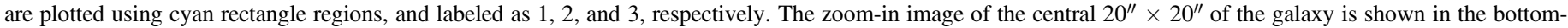
right panel. " $\times$ " marks the sources detected within a 20 " circular radius of the AGN in the Chandra 2-8 keV band image.

ACIS-S detector as part of the Chandra HRC-GTO program (2014 March 09; PI S. Murray; ObsID 15332). We reprocessed the data to create event files with updated calibration modifications, following standard procedures.

We determined the centroid position of the AGN in the Chandra hard energy band of $2-8 \mathrm{keV}$ using the WAVDETECT tool within CIAO with the threshold parameter set to $1 \times 10^{-7}$. We detected three sources within the central $20^{\prime \prime}$ radius of the galaxy in this energy band (see Figure 1). The brightest source was detected at a position of R.A. $=3: 44: 31.83$, and decl. $=-44: 38: 41.22$, with errors of 0 ". 17 and 0 !" 11 , respectively. This is consistent with the 2MASS and Gemini/T-ReCS (see Section 2.4) positions of the nucleus to within $\sim 1^{\prime \prime}$. Therefore, we adopted this Chandra position as the AGN position.

We extracted the source spectrum using the SPECEXTRACT task in CIAO from a circular region of $20^{\prime \prime}$ radius centered on the detected position of the AGN to match the NuSTAR extraction region. A 50" radius circular aperture was used to extract the background counts from an offset, source-free region. The total net count rate within the $20^{\prime \prime}$ radius extraction region in the $0.5-8 \mathrm{keV}$ band is $5.69 \times 10^{-3}$ counts $\mathrm{s}^{-1}$. The net count rate measured by WAVDETECT for the AGN is $1.39 \times 10^{-3}$ counts $\mathrm{s}^{-1}$ in the $0.5-8 \mathrm{keV}$ band. The two other sources detected within the extraction region are located to the north east (NE) and north west (NW) of the AGN. They do not have counterparts at other wavelengths, and are likely to be X-ray binaries within NGC 1448 (see Section 3.2.1). These sources have $0.5-8 \mathrm{keV}$ count rates of $6.67 \times 10^{-4}$ counts $\mathrm{s}^{-1}$ and $8.61 \times 10^{-4}$ counts $\mathrm{s}^{-1}$, for the NE and NW sources, respectively, as measured by WAVDETECT.

\subsection{NTT/EFOSC2}

At optical wavelengths, we performed spectroscopy for NGC 1448 using the ESO NTT on 2015 December 07, using the Faint Object Spectrograph and Camera v.2 (EFOSC2) instrument (Program ID 096.B-0947(A); PI G. B. Lansbury). The source was observed for four $5 \mathrm{~min}$ exposures centered on the Chandra position (see Section 2.2). The slit adopted was 1" $(75 \mathrm{pc})$ in width, and the adopted grism yielded a spectral coverage of 4085-7520 , and spectral resolution of $\mathrm{FWHM}=12.6 \AA$. The seeing at the time of the observation was $\sim 0$."7. Standard IRAF routines were followed to reduce the spectra, and spectrophotometric standard star observations from the same night were used for calibration. As shown in Figure 2, we extracted spectra from three different apertures along the slit, corresponding to: (1) the AGN (using the Chandra position as a reference); (2) the "optical peak"; and (3) the total galaxy. The optical peak corresponds to the position where the optical emission is the brightest in the imaging data and in the two-dimensional spectra, and is offset from the AGN position by $\sim 3^{\prime \prime}$ (R.A. $=3: 44: 31.99$ and decl. $=-44: 38: 42.33)$. The spatial apertures adopted were 2 ". 4 for the AGN and the optical peak, and 36".9 for the total galaxy (see Figure 2). The apertures for the AGN and the optical peak correspond to two spatially distinct, prominent line-emitting regions which are separated by a dust lane, from which we do not see any prominent line emission. The aperture extents for the AGN and the optical peak were chosen based on the light profile of the $\mathrm{H} \alpha$ and $[\mathrm{O}$ III] $\lambda 5007 \AA$ emission lines in the spatial direction, to ensure that we included the total emission of these lines.

Before we can measure the fluxes of the emission lines, we first need to subtract stellar emission from the extracted spectra. 
This was done by fitting the emission line-free regions of each spectrum with a combination of a small subset of galaxy template spectra from the Bruzual \& Charlot (2003) stellar library. This library consists of model templates for 39 stellar populations with ages between $5.0 \times 10^{6}$ and $1.2 \times 10^{10}$ years, and metallicities between 0.008 and 0.05 , with a spectral sampling of $3 \AA$. This library has been used to fit the continua and measure the emission line fluxes of the Sloan Digital Sky Survey galaxy spectra (Tremonti et al. 2004). We performed our spectral synthesis for the three spectra in XSPEC $\mathrm{v} 12.8 .2{ }^{28}$ assuming $z=0.00390$, solar metallicity $\left(Z_{\odot}=0.02\right)$, and the Cardelli et al. (1989) extinction law. The best-fitted stellar spectra were then subtracted from the observed spectra, which resulted in residual spectra with emission lines only. We then analyzed the detected emission lines using the SPLOT tasks in IRAF.

\subsection{Gemini/T-ReCS}

NGC 1448 was observed at MIR wavelengths in 2010 with high spatial resolution using the Thermal-Region Camera Spectrograph (T-ReCS; field of view 28 "! $8 \times 21$ "! 6 ; 0.09 arcsec pixel $^{-1}$; Telesco et al. 1998), mounted on the GeminiSouth telescope. The observation was carried out on 2010 August 19 (Program ID GS-2010B-Q-3) for $\approx 319$ s on-source time using the $N$-band filter $(\lambda=7.4-13.4 \mu \mathrm{m})$ in parallel chop and nod mode. The data were reduced using the MIDIR pipeline in IRAF provided by the Gemini Observatory, and the image analysis was performed using the IDL package MIRPHOT, following Asmus et al. (2014). The data were flux-calibrated with a standard star, HD22663, which was observed immediately before NGC 1448. The resolution of the observation is $\sim 0$ " 4 as measured from the FWHM of the standard star.

A compact nucleus is clearly detected in the MIR continuum at position R.A. $=3: 44: 31.75$ and decl. $=-44: 38: 41.8$ (telescope astrometric uncertainty is on the order of $1^{\prime \prime}$ ) with a $\mathrm{S} / \mathrm{N} \sim 5$. The source appears to be embedded in $\sim 0.7$ extended emission along the host major axis (position angle PA $\sim 44^{\circ}$ ). However, owing to the small extent and often unstable point-spread function (PSF) in ground-based MIR observations, a second epoch of high spatial resolution MIR images is required in order to confirm this extension. The source is best-fitted with a two-dimensional Gaussian model (see Figure 3), which measured a flux density of $f_{12 \mu \mathrm{m}}=12.5 \pm 2.3 \mathrm{mJy}$.

\section{X-Ray Spectral Fitting \\ 3.1. Basic Characterization}

In this section, we describe the broadband X-ray spectral analysis of the AGN in NGC 1448, performed using XSPEC. Given the non-negligible contribution of the background flux to the weak source flux, particularly in the NuSTAR data at high energies, we binned both the Chandra and NuSTAR spectra to a minimum of 1 count per bin and optimized the fitting parameters using the Poisson statistic, C-statistic (Cash 1979). ${ }^{29}$ We included a fixed Galactic absorption component, $N_{\mathrm{H}}^{\mathrm{Gal}}=9.81 \times 10^{19} \mathrm{~cm}^{-2}$ (Kalberla et al. 2005), using the

\footnotetext{
${ }^{28}$ The XSPEC manual is available at http://heasarc.gsfc.nasa.gov/xanadu/ Xspec/XspecManual.pdf.

${ }^{29}$ We note that grouping the Chandra and NuSTAR data to a minimum of 20 and 40 counts per bin, respectively, and performing the spectral fits using $\chi^{2}$ minimization, yields consistent results with the C-statistic approach.
}

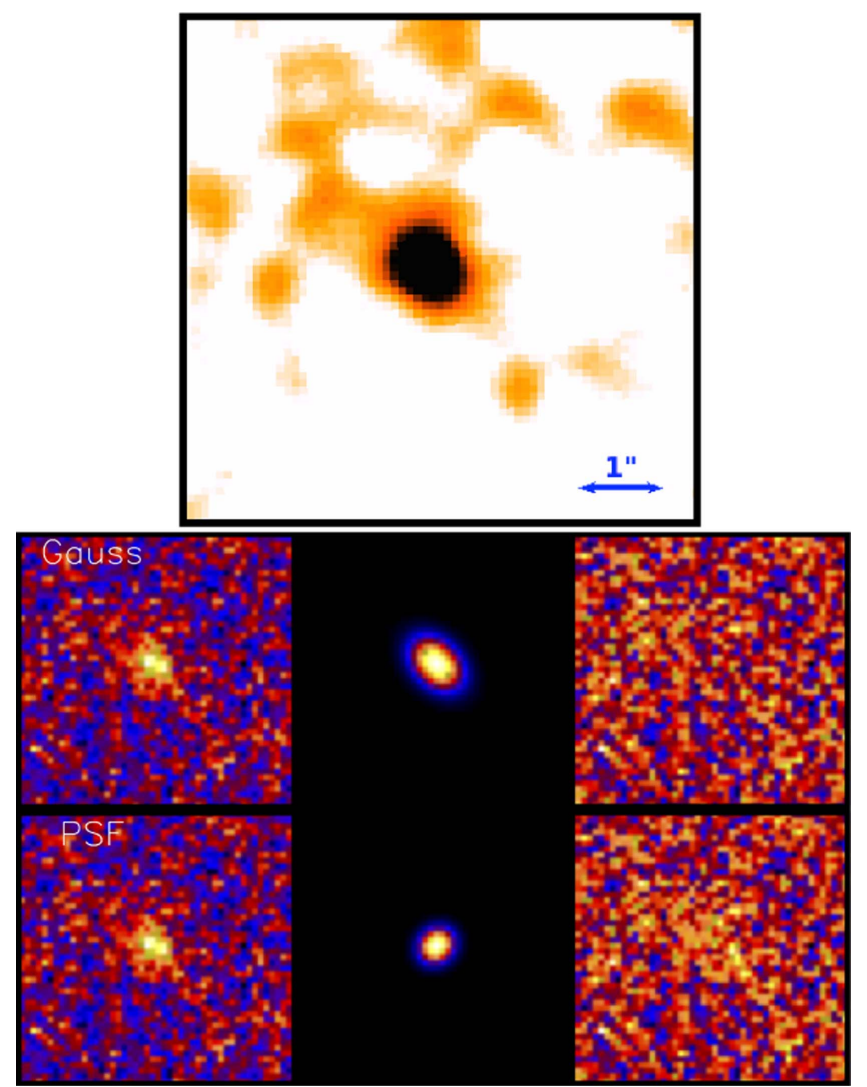

Figure 3. Top: high spatial resolution MIR image of NGC 1448 taken by Gemini/T-ReCS. Bottom: image fits performed using the MIRPHOT task in IDL following Asmus et al. (2014). Shown are the central 4!. $5 \times 4$ !" 5 of the image. The left column is the original image, the middle column is the fit performed on the data, and the right column is the residual after subtracting the fit from the data. The top row shows the results from a Gaussian fit to the total emission, and the bottom row shows the results from fitting the standard star (as a PSF reference) to the total emission. In all images, north is up and east is to the left.

XSPEC model "PHABS" in all spectral fits, and assumed solar abundances for all models. The redshift was fixed at $z=0.00390$ in all analyses. We quoted all errors at $90 \%$ confidence, unless stated otherwise. We summarize the main results of the analysis in Table 1, and present the X-ray data and best-fitting models in Figure 4.

We began our spectral modeling by examining the NuSTAR and Chandra data separately. At this point we are not yet considering non-AGN contributions to the X-ray spectrum, such as from the two off-nuclear point sources within the extraction region. We modeled the two spectra in the $3-40 \mathrm{keV}$ and $2-8 \mathrm{keV}$ bands, respectively, using a simple power-law model with Galactic absorption. The best fitting photon indices are very flat: $\Gamma=-0.29 \pm 0.38$ for NuSTAR (C-stat/dof $=229 / 237)$, and $\Gamma=0.82_{-0.77}^{+0.88}$ for Chandra (C-stat/dof $=125 / 126)$. A slight excess of counts just above $6 \mathrm{keV}$ was observed in both the NUSTAR and Chandra spectra. This is likely an indication of a fluorescent $\mathrm{Fe}-\mathrm{K} \alpha$ line emission, associated with a spectral component produced by AGN emission that is being reflected off a high column of gas. The fluxes of the two spectra within the common energy range of $3-8 \mathrm{keV}$ measured by the model are $f_{3-8}=2.00_{-0.57}^{+0.23} \times 10^{-14} \mathrm{erg} \mathrm{s}^{-1} \mathrm{~cm}^{-2}$ for NuSTAR, and $f_{3-8}=3.50_{-1.34}^{+0.56} \times 10^{-14} \mathrm{erg} \mathrm{s}^{-1} \mathrm{~cm}^{-2}$ for Chandra. These 
Table 1

X-Ray Spectral Fitting Results for NGC 1448

\begin{tabular}{|c|c|c|c|c|}
\hline $\begin{array}{l}\text { Component } \\
\text { (1) }\end{array}$ & $\begin{array}{l}\text { Parameter } \\
(2)\end{array}$ & $\begin{array}{l}\text { Units } \\
(3)\end{array}$ & $\begin{array}{l}\text { Model T } \\
\text { (4) }\end{array}$ & $\begin{array}{l}\text { Model M } \\
(5)\end{array}$ \\
\hline APEC & $k T$ & $\mathrm{keV}$ & $0.63_{-0.33}^{+0.14}$ & $0.63_{-0.42}^{+0.14}$ \\
\hline \multirow[t]{4}{*}{ Absorber/Reflector } & $N_{\mathrm{H}}(\mathrm{eq})$ & $10^{24} \mathrm{~cm}^{-2}$ & $4.2_{-1.7}^{+u}$ & $4.9_{-2.0}^{+u}$ \\
\hline & $N_{\mathrm{H}}(\operatorname{los})$ & $10^{24} \mathrm{~cm}^{-2}$ & $4.2_{-1.7}^{+u}$ & $4.5_{-1.8}^{+u}$ \\
\hline & $\theta_{\text {inc }}$ & $\operatorname{deg}$ & $87^{\mathrm{f}}$ & $78.6_{-11.8}^{+6.5}$ \\
\hline & $\theta_{\text {tor }}$ & $\operatorname{deg}$ & $45.9_{-18.9}^{+33.1}$ & $60^{f}$ \\
\hline \multirow[t]{8}{*}{ AGN Continuum } & $f_{\text {scatt }}$ & $\%$ & $0.2_{-0.2}^{+0.3}$ & $0.1_{-0.1}^{+0.2}$ \\
\hline & $\Gamma$ & $\ldots$ & $1.9^{\mathrm{f}}$ & $1.9^{\mathrm{f}}$ \\
\hline & $L_{0.5-2, \mathrm{obs}}$ & $10^{39} \mathrm{erg} \mathrm{s}^{-1}$ & 0.2 & 0.2 \\
\hline & $L_{2-10, \mathrm{obs}}$ & $10^{39} \mathrm{erg} \mathrm{s}^{-1}$ & 0.9 & 0.9 \\
\hline & $L_{10-40, \text { obs }}$ & $10^{39} \mathrm{erg} \mathrm{s}^{-1}$ & 11.7 & 11.8 \\
\hline & $L_{0.5-2, \text { int }}$ & $10^{40}$ erg s$^{-1}$ & 2.6 & 5.6 \\
\hline & $L_{2-10, \text { int }}$ & $10^{40} \mathrm{erg} \mathrm{s}^{-1}$ & 3.5 & 7.6 \\
\hline & $L_{10-40, \text { int }}$ & $10^{40} \mathrm{erg} \mathrm{s}^{-1}$ & 3.5 & 7.6 \\
\hline C-stat/dof & $\cdots$ & $\cdots$ & $431 / 445$ & $429 / 440$ \\
\hline
\end{tabular}

Note. (1) Model component; (2) parameter associated with each component; (3) units of each parameter; (4) best-fitting parameters for Model T (TORUS model by Brightman \& Nandra 2011); (5) best-fitting parameters for Model M (MYTORUS model by Murphy \& Yaqoob 2009). "f" is a fixed parameter and "u" is an unconstrained parameter. Details of each model are described in Section 3 .

fluxes are consistent with each other within the measurement uncertainties, indicating that there was no significant variability between the two observations.

We then fitted the NUSTAR spectrum simultaneously with the Chandra spectrum between 3 and $40 \mathrm{keV}$ using a simple power-law model and a Gaussian component to model the possible $\mathrm{Fe}-\mathrm{K} \alpha$ emission line, with the line energy and width fixed to $E=6.4 \mathrm{keV}$ and $\sigma=10 \mathrm{eV}$, respectively. This model measured a photon index of $\Gamma=0.02_{-0.28}^{+0.27}$ and $\mathrm{Fe}-\mathrm{K} \alpha$ line equivalent width of $\mathrm{EW}=2.1_{-0.8}^{+1.0} \mathrm{keV}$ (C-stat $/$ dof $=315$ / 331). The flat photon index and large EW measured for the Fe$\mathrm{K} \alpha$ line $(\mathrm{EW} \gtrsim 1 \mathrm{keV})$, are characteristic signatures of $\mathrm{CT}$ absorption.

To test for this, we model both spectra simultaneously from $3-40 \mathrm{keV}$ with the PEXRAV model (Magdziarz \& Zdziarski 1995), which has historically been used to model reflection-dominated AGN spectra. This model produces an AGN continuum which is reflected from a slab-geometry torus with an infinite column density, which is highly unlikely to represent the actual AGN torus. However, it can provide a useful initial test to investigate the AGN spectrum for CT obscuration. Because it does not self-consistently model the fluorescence emission lines expected from a CTAGN, we included a Gaussian component in the model to simulate $\mathrm{Fe}$ $\mathrm{K} \alpha$ narrow line emission, which is the most prominent line produced by CTAGNs. The centroid energy and width of the line were fixed to $E=6.4 \mathrm{keV}$ and $\sigma=10 \mathrm{eV}$, respectively. Due to the limited number of counts, the inclination of the reflector was fixed to the default value set by the model; i.e., $\theta_{\text {inc }} \approx 63^{\circ}$. However, we note that fixing $\theta_{\text {inc }}$ to other values (e.g., near the lower and upper limits of the model; $\theta_{\text {inc }} \approx 26^{\circ}$ and $84^{\circ}$, respectively) has insignificant effects on the parameters obtained. We also fixed the reflection scaling factor to $R=-1$, to simulate a pure reflection spectrum. In addition, we included the absorbed transmitted component of the AGN, simulating the Compton scattering and photoelectric absorption by the torus using CABS and ZPHABS models, respectively. This model provides a decent fit to the data $(\mathrm{C}$-stat $/$ dof $=$ $318 / 330)$, and measured a column density of $N_{\mathrm{H}}(\operatorname{los})=$ $2.7_{-1.2}^{+u} \times 10^{24} \mathrm{~cm}^{-2}$, consistent with CT column. ${ }^{30}$ The intrinsic photon index inferred from the best-fit model is $\Gamma=1.57 \pm 0.35$, in agreement with the typical intrinsic value for an AGN (e.g., Burlon et al. 2011; Corral et al. 2011). The reflection component of the model dominates the transmitted component at all spectral energies probed, indicating that the spectra are reflection-dominated, consistent with being heavily obscured.

\subsection{Physical Modeling}

We proceeded to model the X-ray broadband spectrum of the potentially heavily obscured AGN in NGC 1448 using more physically motivated obscuration models to better characterize the broadband spectrum. The two models used are the TORUS model by Brightman \& Nandra (2011) (Model T), and MYTORUS model by Murphy \& Yaqoob (2009) (Model M). Details and results of the two models are described in Sections 3.2.2 and 3.2.3, respectively. In addition to these models, we added extra components required to account for non-AGN contributions to the X-ray spectrum, and to provide a good fit to the data as described below, and in Section 3.2.1.

At low energies, typically at $E \lesssim 2 \mathrm{keV}$, where the direct emission from a heavily obscured AGN is completely absorbed, other processes can dominate. These processes include X-ray emission radiated by unresolved off-nuclear $\mathrm{X}$-ray sources, thermal emission from a hot interstellar medium, gas photoionized by the $\mathrm{AGN}$, and scattered emission from the AGN. Our data are not of sufficient quality to accurately distinguish between these different physical processes. Therefore, we simply parametrized the low energy part of the spectrum covered by Chandra using the thermal model "APEC" (Smith et al. 2001), ${ }^{31}$ and a power-law component to simulate the scattered emission from the AGN.

A constant parameter $C$ is often included when analyzing data from multiple X-ray observatories simultaneously to account for cross-calibration uncertainties between the various instruments, and to account for any significant variability of the targets between the observations. As detailed earlier, we do not find any significant differences between the Chandra and $N U S T A R$ spectra, indicating that there has not been significant variability between the two observations. Based on the NUSTAR calibration paper (Madsen et al. 2015), the crosscalibration uncertainty of Chandra with respect to NuSTAR is $\approx 1.1$. We therefore decided to fix this parameter to the value found by Madsen et al. (2015) as a conservative approach. However, we note that allowing this parameter to vary in all models returns results that are consistent with this value within statistical uncertainties.

Our two models are described in XSPEC as follows:

$$
\begin{aligned}
\text { Model } \mathrm{T}= & \text { CONSTANT } * \text { PHABS } *(\text { APEC } \\
& + \text { CONS } * \text { ZPOW }+2 \times \text { TBABS } * \text { ZPOW } \\
& + \text { TORUS }),
\end{aligned}
$$

\footnotetext{
30 " $u$ " is unconstrained.

31 We note that the APEC component is mainly contributed by the unresolved emission within the $20^{\prime \prime}$ radius extraction region.
} 

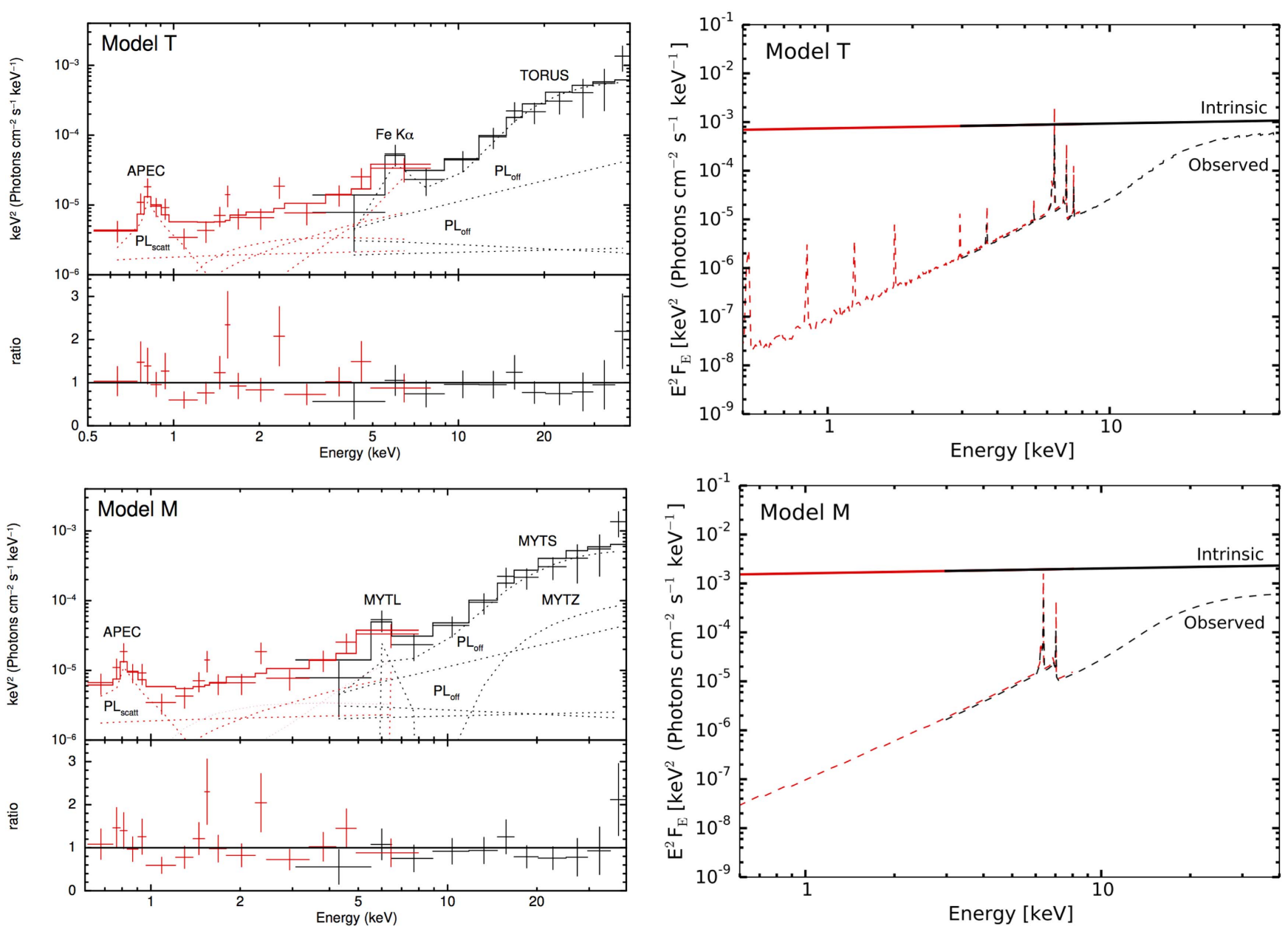

Figure 4. Best-fitting models to the combined NuSTAR and Chandra data of NGC 1448-Model T (top) and Model M (bottom). Model T was fitted between 0.5 and $40 \mathrm{keV}$, and Model $\mathrm{M}$ was fitted between 0.6 and $40 \mathrm{keV}$ due to the strong residuals found at $\sim 0.5 \mathrm{keV}$ for this model. The data have been rebinned to a minimum of $3 \sigma$ significance with a maximum of 25 and 100 bins for NuSTAR and Chandra, respectively, for visual clarity. Color scheme: black (NuSTAR FPMA+B), red (Chandra). Plots on the left show the model components fitted to the data (dotted lines), with the total model shown in solid lines. We included an APEC component and a scattered power-law component to model the emission at the softest energies, and two power-law components to model the two off-nuclear sources located within the extraction region in all models. The iron line modeled in Model $\mathrm{T}$ is labeled as "Fe-K $\alpha$," and the direct, scattered and line components of Model $\mathrm{M}$ are labeled as MYTZ, MYTS, and MYTL, respectively. The top panels of the left plots show the data and unfolded model in $E^{2} F_{E}$ units, while the bottom panels show the ratio between the data and the folded model. Plots on the right show the observed (dashed lines) and intrinsic spectra (solid line) of the AGN component for each model. The slight offsets between the red and black lines are due to the cross-calibration uncertainties between Chandra and NuSTAR (Madsen et al. 2015). These plots show that even at $E \geqslant 10 \mathrm{keV}$, the spectra that we observed for CTAGNs are still significantly suppressed (up to $\sim 2$ orders of magnitude for the case of NGC 1448), demonstrating the extreme of CT absorption.

$$
\begin{aligned}
\text { Model } \mathrm{M}= & \text { CONSTANT } * \text { PHABS } *(\text { APEC } \\
& + \text { CONS } * \text { ZPOW }+2 \times \text { TBABS } * \text { ZPOW } \\
& + \text { ZPOW } * \text { MYTZ }+ \text { MYTS }+ \text { MYTL }) .
\end{aligned}
$$

The two additional absorbed power-law components (TBABS $*$ ZPOW) are included in the models to take into account the two off-nuclear sources detected within the extraction region in the Chandra data. We discuss these sources in the following section.

\subsubsection{Off-nuclear X-Ray Sources}

In this section, we present an analysis of the two off-nuclear point sources detected in the $0.5-8 \mathrm{keV}$ Chandra band within the $20^{\prime \prime}$ radius extraction region (see Figure 1). We extracted their spectra using extraction regions of $3^{\prime \prime}$ and $2^{\prime \prime}$ for the NE and NW sources, respectively. Due to limited counts $(\sim 30$ counts at $0.5-8 \mathrm{keV}$ for each source), we binned their spectra to a minimum of 5 counts per bin and optimized the fitting parameters using $\mathrm{C}$-statistic. We model both sources using a simple power-law model absorbed by host galaxy absorption (TBABS), in addition to the Galactic absorption.

The photon indices and intrinsic absorption columns measured by the fits are $\Gamma=1.02_{-1.28}^{+1.84}$ and $N_{\mathrm{H}}$ (int) $\leqslant$ $2.19 \times 10^{22} \mathrm{~cm}^{-2}(\mathrm{C}$-stat/dof $=8.1 / 7)$ for the NE source, and $\Gamma=2.15_{-1.30}^{+2.03}$ and $N_{\mathrm{H}}$ (int) $\leqslant 2.11 \times 10^{22} \mathrm{~cm}^{-2}$ (C-stat/ dof $=7.4 / 10$ ) for the NW source. The $0.5-8 \mathrm{keV}$ intrinsic luminosities for both sources, assuming that they are located within NGC 1448, are $2.23_{-0.85}^{+1.83} \times 10^{38} \mathrm{erg} \mathrm{s}^{-1}$ and $1.56_{-0.09}^{+1.77} \times 10^{38} \mathrm{erg} \mathrm{s}^{-1}$ for the NE and NW sources, respectively. These luminosities are almost an order of magnitude lower than the threshold luminosity for ultraluminous X-ray sources $\left(L_{\mathrm{X}} \gtrsim 10^{39} \mathrm{erg} \mathrm{s}^{-1}\right)$.

The best-fitted photon index for the NE source is potentially flat (although with large uncertainties), and may suggest that it is a background obscured AGN. We estimate the probability of finding an AGN within a random $20^{\prime \prime}$ radius region with at least the observed flux of the NW source $\left(f_{0.5-8} \gtrsim\right.$ $4 \times 10^{-15} \mathrm{erg} \mathrm{s}^{-1} \mathrm{~cm}^{-2}$ ) using the AGN number counts of 
the $4 \mathrm{Ms}$ Chandra Deep Field South Survey (Lehmer et al. 2012). Based on this, the probability of finding two or more background AGNs within a $20^{\prime \prime}$ radius circular region is $<1 \%$. In addition, we do not find counterparts to these Chandra sources at other wavelengths (e.g., MIR and optical). Given their high Galactic latitudes $\left(|b| \sim 51^{\circ}\right)$, it is unlikely that these are Galactic sources. Based on these arguments, we conclude that the two off-nuclear X-ray sources are more likely to be X-ray binaries within NGC 1448. Given the stellar mass and SFR of the galaxy, we would expect to find $\sim 3 \mathrm{X}$-ray binaries with $L_{0.5-8}>10^{38} \mathrm{erg} \mathrm{s}^{-1}$ in NGC 1448 (Gilfanov 2004; Mineo et al. 2012). This is consistent with our detections of two potential candidates within the central $20^{\prime \prime}$ circular radius of the galaxy. We therefore included the two absorbed power-law components detailed above into Model T and $\mathrm{M}$ to account for their maximum contributions to our X-ray spectra of NGC 1448. We fixed all the parameters at the best-fit values measured from the Chandra data.

Due to the NuSTAR PSF, sources that lie outside the $20^{\prime \prime}$ radius extraction region could also contaminate our spectrum. We tested whether these sources contribute a significant fraction of our X-ray broadband spectrum by including the best-fit power-law model of the source $\sim 30^{\prime \prime}$ west of the AGN to our X-ray spectral modeling of NGC 1448. This source is the brightest in a $50^{\prime \prime}$ radius circular region around the AGN which corresponds to a $\sim 70 \% \mathrm{NUSTAR}$ ECF. We left the normalization of the source component free to vary, and found that the value is consistent with zero, suggesting that the source does not significantly contaminate our broadband AGN spectrum. We therefore did not include this source, and consequently any of the fainter sources outside the extraction region, in our modeling of the AGN X-ray spectra.

\subsubsection{Model T}

The TORUS model by Brightman \& Nandra (2011) (Model T) simulates obscuration by a spherical torus with variable biconical polar opening angle $\left(\theta_{\text {tor }}\right)$ ranging between $26-84^{\circ}$. The line-of-sight column density, $N_{\mathrm{H}}(\mathrm{los})$, through the torus, which is equal to the equatorial column density $N_{\mathrm{H}}(\mathrm{eq})$, is independent of the inclination angle $\left(\theta_{\text {inc }}\right)$, and extends up to $10^{26} \mathrm{~cm}^{-2}$, an order of magnitude higher than that allowed by the MYTORUS model (see Section 3.2.3). The model also selfconsistently predicts the Compton scattering and fluorescent $\mathrm{Fe}-\mathrm{K} \alpha$ and $\mathrm{Fe}-\mathrm{K} \beta$ lines, as well as $\mathrm{K} \alpha$ emission from $\mathrm{C}, \mathrm{O}, \mathrm{Ne}$, $\mathrm{Mg}, \mathrm{Si}, \mathrm{Ar}, \mathrm{Ca}, \mathrm{Cr}$, and $\mathrm{Ni}$, which are commonly seen in CTAGNs. The model is available between 0.1 and $320 \mathrm{keV}$.

In addition to the TORUS model, we included several other model components as described earlier in Sections 3 and 3.2.1 to provide a good fit to the data. Initially, we left both $\theta_{\text {inc }}$ and $\theta_{\text {tor }}$ free to vary; however, they were both unconstrained. We then fixed the torus inclination angle to the upper limit of the model $\left(\theta_{\text {inc }}=87^{\circ}\right)$, to simulate an edge-on inclination torus, and to allow for the full exploration of $\theta_{\text {tor }}$ (Brightman et al. 2015). The column density measurement is insensitive to $\theta_{\text {inc }}$ when $\theta_{\text {inc }}>\theta_{\text {tor }}$ (Brightman et al. 2015), and therefore should not significantly affect the $N_{\mathrm{H}}(\mathrm{los})$ measured. In addition, we fixed the photon index to $\Gamma=1.9$ as it could not be constrained simultaneously with $\theta_{\text {tor }}$.

The model implied a column density of $N_{\mathrm{H}}(\mathrm{los})=$ $4.2_{-1.7}^{+u} \times 10^{24} \mathrm{~cm}^{-2}(\mathrm{C}$-stat $/$ dof $=431 / 445)$. The lower limit of this column density is well above the CT threshold, and therefore this model confirms that the AGN is CT. The best-fit torus opening angle is $\theta_{\text {tor }}=45.9_{-18.9}^{+33.1}$ degrees, suggesting a geometrically thick torus. The model measured a small scattering fraction of $0.2_{-0.2}^{+0.3} \%$ with respect to the intrinsic power-law. This is consistent with that found in other obscured AGN (e.g., Noguchi et al. 2010; Gandhi et al. 2014, 2015). However we note that given the modest quality of our data, the scattered power-law component will also include contributions from other processes such as unresolved X-ray binaries. Therefore, the true AGN scattering fraction could be lower than this value. We inferred the intrinsic luminosities of the AGN in three different bands based upon the best-fit parameters obtained, as presented in Table 1.

\subsubsection{Model $M$}

The MYTORUS model by Murphy \& Yaqoob (2009) (Model M) simulates a toroidal absorber geometry with a fixed opening angle of $\theta_{\text {tor }}=60^{\circ}$ and variable inclination angle. The line-ofsight column density, $N_{\mathrm{H}}(\mathrm{los})$, is derived from the measured inclination angle and equatorial column density, $N_{\mathrm{H}}(\mathrm{eq})$, which is simulated only up to a column density of $N_{\mathrm{H}}(\mathrm{eq})=10^{25}$ $\mathrm{cm}^{-2}$. There is more freedom in exploring complex absorbing geometry in the MYTORUS model as it allows the user to disentangle the direct (MYTZ), scattered (MYTS), and lineemission (MYTL) components from each other. The MYTL component simulates the neutral $\mathrm{Fe}-\mathrm{K} \alpha$ and $\mathrm{Fe}-\mathrm{K} \beta$ fluorescence lines, and the associated Compton shoulders for the AGN. The model is defined between 0.5 and $500 \mathrm{keV}$. However, we noticed strong residuals at $\sim 0.5 \mathrm{keV}$ which could be attributed to the fact that we are probing the lower limit of the model. Therefore for this model, we restricted our fit to above $0.6 \mathrm{keV}$.

For simplicity, we fitted the AGN spectrum by coupling all the parameters of the scattered and fluorescent line components to the direct continuum component. The relative normalizations of MYTS $\left(A_{S}\right)$ and MYTL $\left(A_{L}\right)$ with respect to MYTZ $\left(A_{Z}\right)$ were set to 1 . At first, we left the photon index free to vary; however, it reached the lower limit of the model, suggesting that it is not well constrained. Therefore, we fixed the value of this parameter to $\Gamma=1.9$.

Model $\mathrm{M}$ gives as good a fit to the data as Model $\mathrm{T}$, with $\mathrm{C}$-Stat $/$ dof $=429 / 440$. Using this model, we measured an equatorial column density of $N_{\mathrm{H}}(\mathrm{eq})=4.9_{-2.0}^{+u} \times 10^{24} \mathrm{~cm}^{-2}$. The model measured a high inclination angle of $\theta_{\text {inc }}=$ $78.6_{-11.8}^{+6.5}$ degrees, close to the maximum value fixed in Model T. The corresponding $N_{\mathrm{H}}(\mathrm{los})$ value is well within the CT regime, within the uncertainties, $N_{\mathrm{H}}(\operatorname{los})=$ $4.5_{-1.8}^{+u} \times 10^{24} \mathrm{~cm}^{-2}$, and agrees very well with that measured by Model T. ${ }^{32}$ This model also measured a small scattering fraction; i.e., $0.1_{-0.1}^{+0.2} \%$, consistent with Model T. As with Model T, we determined the intrinsic luminosity of the AGN in different bands from the best-fitting parameters (see Table 1).

\section{Multiwavelength Results}

In this section, we present the results from the optical and MIR observations of NGC 1448 as detailed in Sections 2.3 and 2.4 , respectively. We use these data to calculate the properties of the AGN at different wavelengths to compare with the results of our X-ray spectral analysis.

\footnotetext{
32 The line-of-sight column density for Model M was derived using Equation (3.1) of Murphy \& Yaqoob (2009), and assuming an inclination angle at the best-fit value.
} 


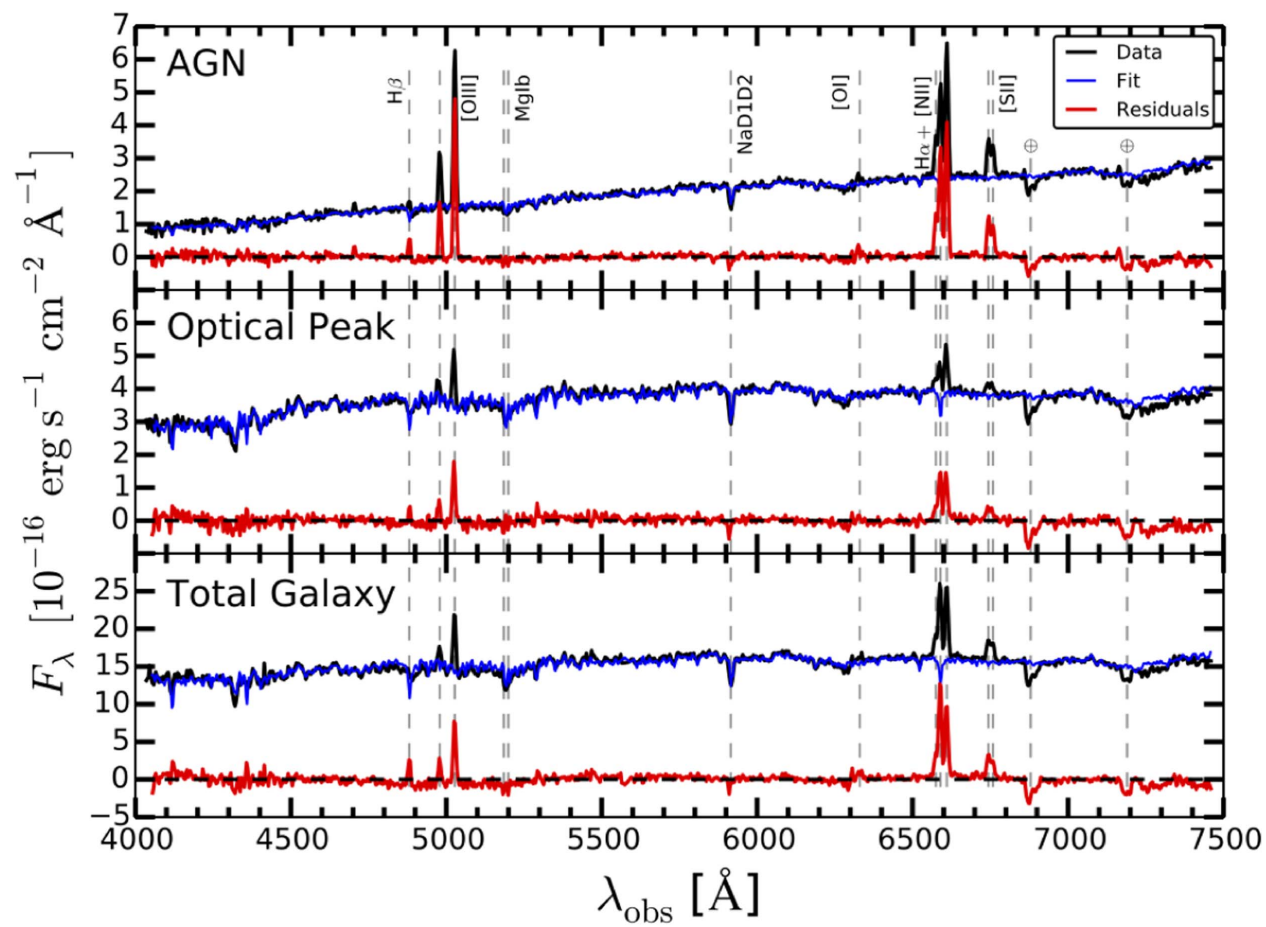

Figure 5. The optical spectra for the AGN (top), optical peak (middle), and the whole galaxy (bottom) extracted from the aperture regions shown in Figure 2. Black are the observed spectra, blue are the best-fitted stellar template spectra, and red are the residual spectra obtained after subtracting the best-fitted stellar template spectra from the observed spectra. Detected emission and absorption lines are labeled with dotted lines. The absorption lines labeled with $\oplus$ are telluric.

\subsection{Optical}

As described in Section 2.3, we extracted optical spectra from three different regions along the slit; i.e., the AGN, optical peak, and across the whole galaxy. The extracted spectra, along with the fits obtained from the spectral synthesis modeling (see Section 2.3), and the fit residuals, are shown in Figure 5. As shown in this figure, no significant residuals were left after fitting the observed spectra with stellar population templates, without the need of additional AGN continuum components. This suggests that the AGN optical continuum is highly obscured. Spectral analysis revealed that the stellar emission from all three regions is due to both young $(5 \mathrm{Myr})$ and old (5 Gyr) stellar populations, dominated by the latter with $\sim 75 \%-$ $84 \%$ contributions measured between the three spectra. Although the optical peak is where the total optical emission is the brightest, the emission lines are the strongest at the Chandra position, providing independent evidence of the AGN location within the galaxy.

The interstellar extinction measured toward the stellar population at the AGN position from the spectral synthesis modeling is much higher than at the optical peak position, $A_{V}^{\mathrm{AGN}}=2.15_{-0.06}^{+0.16} \mathrm{mag}$ and $A_{V}^{\text {peak }}=0.59_{-0.05}^{+0.04} \mathrm{mag}$, respectively, further suggesting that the optical emission from the AGN is obscured along our line of sight. The total extinction measured from the whole galaxy spectra, $A_{V}^{\text {gal }}=0.73 \pm$ $0.06 \mathrm{mag}$, is $\sim 3 \times$ lower than that measured at the AGN position, demonstrating that the extinction across the galaxy is non-uniform and inhomogeneous.

We measured the emission line fluxes and ratios for the AGN, optical peak, and the whole galaxy from the extinction-corrected residual spectra (see Table 2). First, we calculated the extinctions toward the AGN narrow line
Table 2

Optical Emission Line Fluxes and Ratios for Spectra Shown in Figure 5

\begin{tabular}{lccc}
\hline \hline Emission Line & AGN & $\begin{array}{c}\text { Optical } \\
\text { Peak }\end{array}$ & $\begin{array}{c}\text { Total } \\
\text { Galaxy }\end{array}$ \\
\hline $\mathrm{H} \alpha$ & $3.79 \pm 0.08$ & $1.51 \pm 0.17$ & $15.2 \pm 0.50$ \\
$\mathrm{H} \beta$ & $0.65 \pm 0.05$ & $0.40 \pm 0.07$ & $3.60 \pm 0.32$ \\
{$[\mathrm{O}$ III $] \lambda 4959$} & $1.85 \pm 0.05$ & $0.74 \pm 0.10$ & $3.51 \pm 0.38$ \\
{$[\mathrm{O}$ III $] \lambda 5007$ (obs.) } & $5.38 \pm 0.06$ & $2.07 \pm 0.09$ & $9.78 \pm 0.37$ \\
{$[\mathrm{O}$ III $] \lambda 5007$ (int.) } & $43.7 \pm 0.49$ & $16.8 \pm 0.73$ & $79.4 \pm 3.00$ \\
{$[\mathrm{O}$ I $] \lambda 6300$} & $0.38 \pm 0.08$ & $<0.34$ & $2.22 \pm 0.60$ \\
{$[\mathrm{~N}$ II $] \lambda 6549$} & $1.51 \pm 0.08$ & $0.58 \pm 0.19$ & $3.44 \pm 0.55$ \\
{$[\mathrm{~N}$ II $] \lambda 6583$} & $4.71 \pm 0.06$ & $1.73 \pm 0.10$ & $11.7 \pm 0.39$ \\
{$[\mathrm{~S} \mathrm{II}] \lambda 6717$} & $1.36 \pm 0.09$ & $0.40 \pm 0.27$ & $3.41 \pm 0.87$ \\
{$[\mathrm{~S} \mathrm{II}] \lambda 6731$} & $1.12 \pm 0.09$ & $0.57 \pm 0.29$ & $3.14 \pm 0.93$ \\
\hline Line Ratio & & & \\
\hline $\mathrm{H} \alpha / \mathrm{H} \beta$ & $5.83 \pm 0.47$ & $3.78 \pm 0.79$ & $4.22 \pm 0.40$ \\
{$[\mathrm{O}$ III $] \lambda 5007 / \mathrm{H} \beta$} & $8.28 \pm 0.64$ & $5.18 \pm 0.93$ & $2.72 \pm 0.26$ \\
{$[\mathrm{O}$ I $] \lambda 6300 / \mathrm{H} \alpha$} & $0.10 \pm 0.02$ & $<0.23$ & $0.15 \pm 0.04$ \\
{$[\mathrm{~N}$ II $] \lambda 6583 / \mathrm{H} \alpha$} & $1.24 \pm 0.03$ & $1.15 \pm 0.15$ & $0.77 \pm 0.04$ \\
{$[\mathrm{~S} \mathrm{II}](\lambda 6717+\lambda 6731) / \mathrm{H} \alpha$} & $0.65 \pm 0.04$ & $0.64 \pm 0.27$ & $0.43 \pm 0.08$ \\
\hline
\end{tabular}

Note. The fluxes are given in units of $10^{-15} \mathrm{erg} \mathrm{s}^{-1} \mathrm{~cm}^{-2}$. The intrinsic [O III] $\lambda 5007$ flux was corrected for the Balmer decrement.

region (NLR) from the Balmer decrements at the three positions. We assumed an intrinsic Balmer decrement of $(\mathrm{H} \alpha / \mathrm{H} \beta)_{\text {int }}=2.86$, which corresponds to a temperature of $T=10^{4} \mathrm{~K}$ and an electron density $n_{e}=10^{2} \mathrm{~cm}^{-3}$ for Case B recombination (Osterbrock 1989). Based on these, we found $A_{V}^{\mathrm{AGN}}=1.89 \pm 0.03 \mathrm{mag}, \quad A_{V}^{\text {peak }}=0.74 \pm 0.09 \mathrm{mag}$, and $A_{V}^{\text {gal }}=1.03 \pm 0.04 \mathrm{mag}$. The high extinction measured 

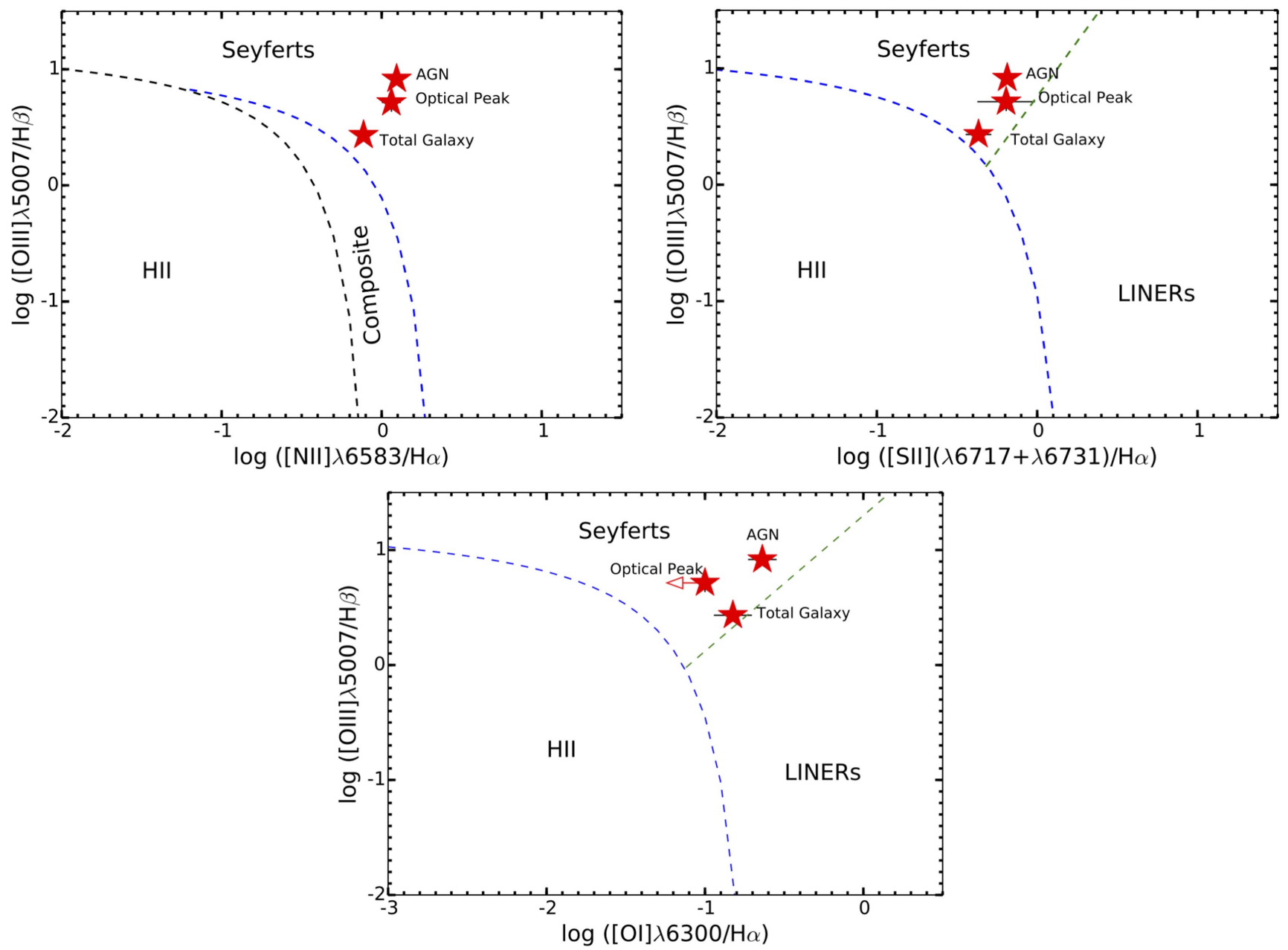

Figure 6. Location of the AGN, optical peak, and the total galaxy in the BPT diagnostic diagrams. The blue dashed lines indicate the maximum limit for the ionization of gas by a starburst found by Kewley et al. (2001), black dashed lines are the empirical division between H II regions and AGNs proposed by Kauffmann et al. (2003), and the green dashed lines are the division between LINERs and Seyfert galaxies, proposed by Kewley et al. (2006). All three regions from our narrow slit spectroscopy show evidence for an AGN in NGC 1448.

at the AGN position provides strong evidence that the AGN is heavily obscured at optical wavelengths by the host galaxy.

Using the emission line ratios tabulated in Table 2, we constructed Baldwin-Philips-Terlevich (BPT) diagnostic diagrams (Baldwin et al. 1981) for NGC 1448. This is shown in Figure 6. Based on these diagrams, all three spectra fall within the region of Seyfert galaxies, with the AGN having slightly higher emission line ratios. These results provide the first identification of the AGN in NGC 1448 at optical wavelengths, and also confirm the position of the AGN in the galaxy. The total galaxy spectrum is located close to the $\mathrm{H} \mathrm{II} /$ low ionization emission line regions (LINERs) of the BPT diagrams. This might explain why the AGN was misidentified as an $\mathrm{H}$ II region in Veron-Cetty \& Veron (1986); i.e., due to host galaxy contaminations as a result of the larger slit size used to extract the spectrum.

The separation between the AGN and the optical peak, $~ 225$ $\mathrm{pc}$, is consistent with the NLR scale observed in AGNs with comparable luminosities as NGC 1448 (Bennert et al. 2006). The apparent separation observed between the two can be attributed to the presence of a dust lane which obscures part of the system along our line of sight.
The intrinsic [O III] $\lambda 5007 \AA$ flux of the AGN corrected for the Balmer decrement is about an order of magnitude higher than the observed luminosity (see Table 2). The [O III] line emission in AGN is mostly produced in the NLR due to photoionization from the central source. Since this region extends beyond the torus, it does not suffer from nuclear obscuration like the X-ray emission. However as we demonstrated earlier, it can suffer from significant optical extinction from the host galaxy. Indeed, in extreme cases, the host galaxy obscuration can be so high that the optical Balmer decrement only provides a lower limit on the extinction (Goulding \& Alexander 2009).

Using our intrinsic [O III] luminosity measured for the AGN, $L_{\text {[OIII] }}=(6.89 \pm 0.08) \times 10^{38} \mathrm{erg} \mathrm{s}^{-1}$, we proceeded to compare the intrinsic X-ray luminosity determined from our X-ray spectral fitting with that predicted from the X-ray:[O III] intrinsic luminosity relationship of Panessa et al. (2006). Based on this correlation, we infer an intrinsic X-ray luminosity of $L_{2-10, \text { int }}=(0.2-4.5) \times 10^{40} \mathrm{erg} \mathrm{s}^{-1}{ }^{33}$ This is consistent with that determined from our X-ray spectral fittings, providing confidence that our analysis is reliable.

\footnotetext{
${ }^{33}$ The given luminosity range accounts for the mean scatter of the correlation.
} 


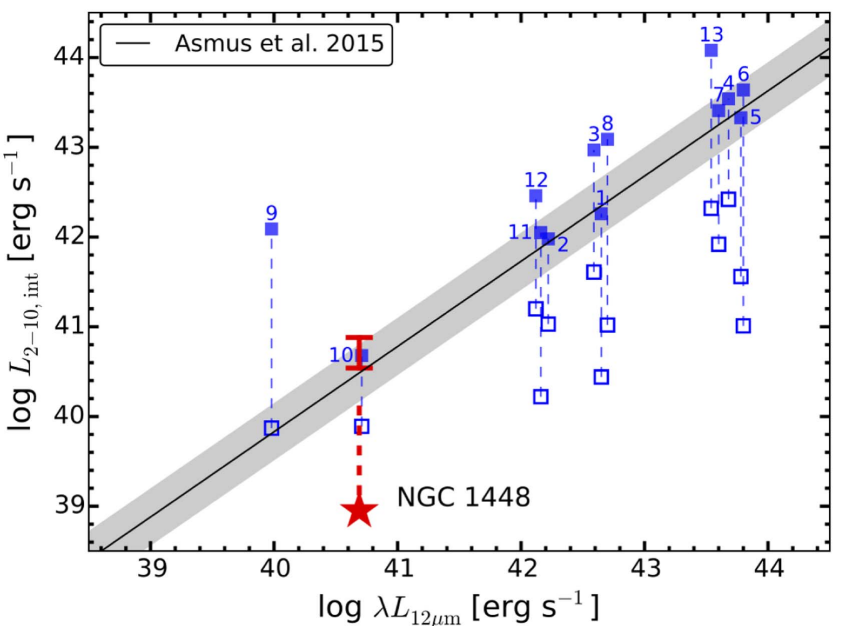

Figure 7. The $2-10 \mathrm{keV}$ vs. $12 \mu \mathrm{m}$ luminosity plot of NGC 1448 . The observed $2-10 \mathrm{keV}$ luminosity is plotted using the red star, and the red solid line marks the range of the intrinsic luminosity measured from our X-ray spectral analysis. The black solid line is the $2-10 \mathrm{keV}$ vs. $12 \mu \mathrm{m}$ luminosity relationship of AGN from Asmus et al. (2015). The shaded region indicates the intrinsic scatter of the relation. The plot shows that our measured intrinsic 2-10 keV luminosity agrees very well with the Asmus et al. (2015) correlation, providing evidence that our spectral analysis reliably characterizes the intrinsic power of the AGN. The observed and intrinsic luminosity of the Gandhi et al. (2014) local bona-fide CTAGNs with high spatial resolution $12 \mu \mathrm{m}$ available from Asmus et al. (2014), are shown as open and filled blue squares, respectively (see footnote 34). As can be seen from this plot, NGC 1448 is consistent with the other bona-fide CTAGNs with typical observed 2-10 keV luminosities that are $\sim 2$ orders of magnitude lower than the Asmus et al. (2015) relation. However, when corrected for absorption, their luminosities are more in agreement with the correlation, with the exception for NGC 4945, which is known to be overluminous in X-rays. The local CTAGNs plotted in the figure are as follows, with the reference for X-ray data given in parentheses if they were not obtained from Asmus et al. (2015): (1) Circinus; (2) ESO5-G4 (Ueda et al. 2007); (3) ESO138-G1; (4) Mrk 3; (5) NGC424; (6) NGC 1068; (7) NGC 3281; (8) NGC 3393; (9) NGC 4945; (10) NGC 5194 (Terashima et al. 1998; Goulding et al. 2012); (11) NGC 5643 (Annuar et al. 2015); (12) NGC 5728; (13) NGC 6240.

\subsection{Mid-infrared}

The high spatial resolution MIR observation by Gemini/T-ReCS measured a $12 \mu \mathrm{m}$ luminosity of $\lambda L_{12 \mu \mathrm{m}}=$ $(4.90 \pm 0.93) \times 10^{40} \mathrm{erg} \mathrm{s}^{-1}$ for NGC 1448 (see Section 2.4). MIR luminosity is predicted to provide an accurate estimate for the intrinsic luminosity of the AGN. This is because the absorbed X-ray radiation from the central engine is mostly reemitted in the MIR by the torus. To further test the absorptioncorrected X-ray luminosity measured from our spectral analysis, we also compared the luminosity measured from our analysis with that predicted from the X-ray:MIR correlation constructed based upon high angular resolution MIR observations of local Seyferts, which has been shown to trace the intrinsic X-ray luminosity of AGNs very well (e.g., Horst et al. 2008; Gandhi et al. 2009; Asmus et al. 2015). The correlation with respect to the recent Asmus et al. (2015) relation is shown in Figure 7. The figure shows that the observed 2-10 keV luminosity of the AGN in NGC 1448 is about two orders of magnitude lower than this relation, similar to other local bona-fide CTAGNs (Gandhi et al. 2014), indicating extreme obscuration. ${ }^{34}$ However, after correcting

\footnotetext{
${ }^{34}$ We note that the bona-fide CTAGN sample (Gandhi et al. 2014) plotted in Figures 7-9 was updated to include a new local bona-fide CTAGN, NGC 5643 (Annuar et al. 2015), and exclude NGC 7582 which has been shown to have variable $N_{\mathrm{H}}$ (Rivers et al. 2015).
}

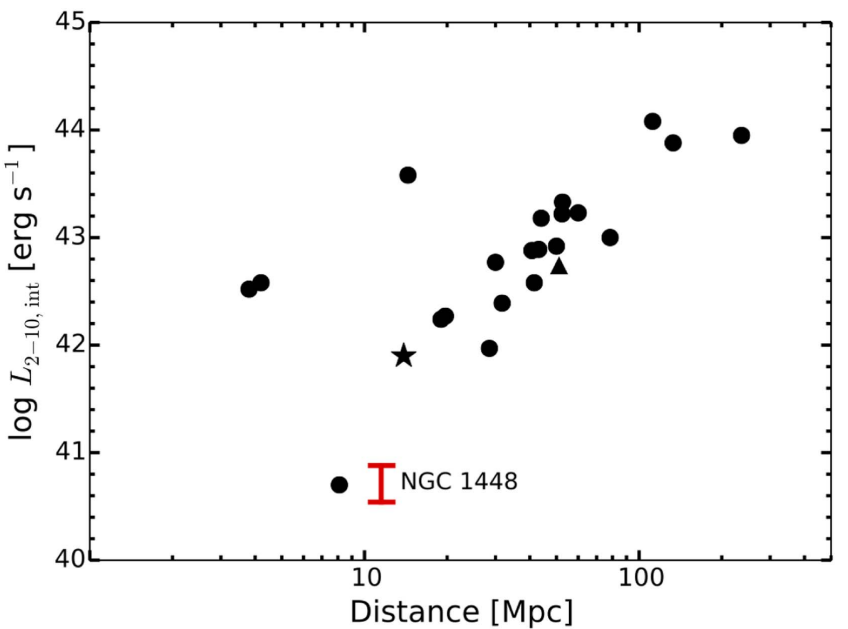

Figure 8. Intrinsic 2-10 keV luminosity vs. distance of local bona-fide CTAGNs (black circles) updated from Gandhi et al. (2014) to include NGC 5643 (black star; Annuar et al. 2015) and NGC 1448 (see footnote 34). Black triangle indicates NGC 4939 which has the lower limit to its $2-10 \mathrm{keV}$ luminosity plotted. The range of intrinsic luminosity of NGC 1448 is plotted in red.

for absorption measured from our X-ray spectral modeling, the luminosity agrees very well with the intrinsic relationship of Asmus et al. (2015), further supporting our analysis.

\section{Discussion}

In this paper, we presented a multiwavelength view of the AGN in NGC 1448 across three wavebands; i.e., the MIR continuum, optical, and X-rays. Our new data provide the first identification of the AGN at these wavelengths. Combining our NUSTAR data with an archival Chandra observation, we performed broadband X-ray spectral analysis $(\approx 0.5-40 \mathrm{keV})$ of the AGN.

We fitted the spectra with two different obscuration models, the TORUS and MYTORUS models (Sections 3.2.2 and 3.2.3, respectively), which fitted the spectra equally well and gave results that are consistent with each other. Considering both models, we found that the AGN is buried under a CT column of obscuring gas, with a column density of $N_{\mathrm{H}}(\mathrm{los}) \gtrsim$ $2.5 \times 10^{24} \mathrm{~cm}^{-2}$. Despite the uncertainties in the parameters and the different geometries simulated by the two models, as well as the phenomenological model simulated by the PEXRAV model discussed in Section 3.1, a CT solution is required to get a best fit to the data in all cases. The $2-10 \mathrm{keV}$ intrinsic luminosity measured from the two physical models ranges between $L_{2-10 \text {,int }}=(3.5-7.6) \times 10^{40} \mathrm{erg} \mathrm{s}^{-1}$, making NGC 1448 one of the lowest luminosity bona-fide CTAGNs in the local universe, comparable to NGC 5194 (Terashima et al. 1998; see Figure 8). The measured column density is so extreme that even at $E>10 \mathrm{keV}$, the observed spectrum is still significantly suppressed (up to $\sim 2$ orders of magnitude lower than the intrinsic spectrum; see Figure 4). This demonstrates why it is very challenging to identify CTAGNs, even at hard X-ray energies, and at this distance. The predicted $14-195 \mathrm{keV}$ flux of the AGN, $f_{14-195} \sim 10^{-12} \mathrm{erg} \mathrm{s}^{-1} \mathrm{~cm}^{-2}$, is an order of magnitude lower than the deepest flux level reached by the Swift-BAT all sky survey $\left(f_{14-195} \sim\right.$ $10^{-11} \mathrm{erg} \mathrm{s}^{-1} \mathrm{~cm}^{-2}$; Baumgartner et al. 2013), demonstrating that pointed observations with $N u S T A R$ have better sensitivity in finding faint CTAGNs. 
We used our measured 2-10 keV intrinsic X-ray luminosities to estimate the Eddington ratio of NGC 1448. Goulding et al. (2010) made an estimation of the supermassive black hole mass ( $M_{\mathrm{BH}}$ ) using the $M_{\mathrm{BH}}$ to bulge $K$-band luminosity relation by Marconi \& Hunt (2003), and inferred $\log \left(M_{\mathrm{BH}} / M_{\odot}\right)=6.0_{-0.5}^{+0.1}$. Based on this black hole mass, we found that the AGN is accreting matter at $\sim 0.6 \%-1.2 \%$ of the Eddington rate. ${ }^{35,36}$

Some studies have suggested that the AGN torus is not developed at low luminosity, $L_{\text {bol }} \lesssim 10^{42} \mathrm{erg} \mathrm{s}^{-1}$, and/or at low mass accretion rate, $\lambda_{\text {Edd }} \lesssim 2 \times 10^{-4}$ (e.g., Elitzur \& Shlosman 2006; Hönig \& Beckert 2007; Kawamuro et al. 2016). The identification of NGC 1448 as a low luminosity CTAGN (as demonstrated from the X-ray spectral fitting and MIR analysis) with a bolometric luminosity potentially below the quoted limit, $L_{\text {bol }} \sim(0.7-1.5) \times 10^{42} \mathrm{erg} \mathrm{s}^{-1}$ however, does not provide clear evidence for the disappearance of the torus at the low AGN luminosities proposed by some studies.

The CTAGN in NGC 1448 is hosted by a nearly edge-on spiral galaxy, which could contribute to at least some of the $\mathrm{X}$-ray obscuration we observed. Our X-ray data are not of sufficient quality to allow us to investigate the origin of the CT obscuration in NGC 1448 in detail. However, our optical spectral synthesis modeling suggests an extinction of $A_{V}=2.15$ toward the AGN along our line of sight, which translates into a column density of $N_{\mathrm{H}}(\mathrm{los})=4.8 \times 10^{21}$ $\mathrm{cm}^{-2}$, assuming the gas-to-dust ratio of the Milky Way (Güver \& Özel 2009). This suggests that the CT column density we measured from the X-ray spectral fittings is not due to the host galaxy material, but is due to the AGN circumnuclear material itself.

High host galaxy inclinations along our line of sight can also affect AGN selection in the local universe as demonstrated by, e.g., Goulding \& Alexander (2009). Material in the host galaxies of highly inclined systems can severely suppress optical emission from AGNs, causing them to be misidentified at optical wavelengths. Figure 9 shows the distribution of host galaxy inclinations for local CTAGNs (adapted from Gandhi et al. 2014; see footnote 34), as compared to the expected distribution from random galaxy orientations. If we are missing CTAGNs in the local universe due to AGN host inclination selection effects, we might expect the local CTAGN sample to be lacking at high host inclinations. However, Figure 9 suggests that this is not the case. Performing a KolmogorovSmirnov (KS) test between the two distributions inferred a KS test probability of $P_{\mathrm{KS}} \sim 0.4$. This indicates that the two distributions are not significantly different from each other, and suggests that the current sample of local CTAGNs is not affected by host galaxy inclinations (see also Koss et al. 2016). Therefore, although the census of CTAGNs is incomplete, even in the local universe (at $D \approx 200 \mathrm{Mpc}$ ), we do not find strong evidence that incompleteness is due to host galaxy inclination.

\section{Summary}

In this paper, we have presented a multiwavelength view of the AGN in NGC 1448 across MIR continuum, optical, and $\mathrm{X}$-rays. Our data provide the first identifications of the AGN at

\footnotetext{
35 We assumed an AGN bolometric correction of $L_{\text {bol }} / L_{2-10}=20$ (e.g., Vasudevan et al. 2010)

${ }^{36}$ The calculation for the Eddington ratio involves highly uncertain quantities, therefore the inferred value is subject to errors of a factor of a few (Brandt \& Alexander 2015).
}

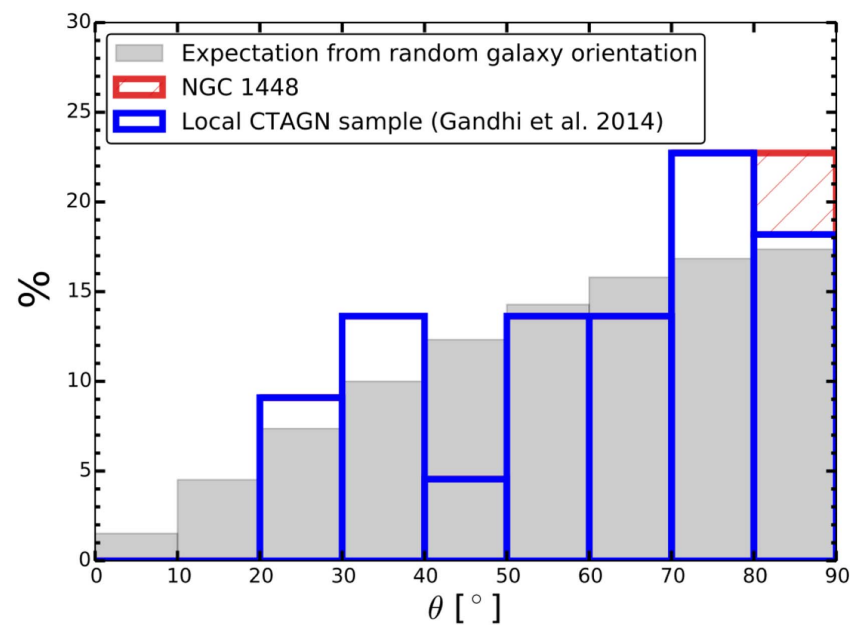

Figure 9. Distribution of host galaxy inclination angles $(\theta)$ for the Gandhi et al. (2014) local CTAGN sample (where available) plotted in blue (see footnote 34). NGC 1448 is plotted in hatched red. The shaded region shows the expected distribution from random galaxy orientation. The total distribution of the local CTAGNs is consistent with the expected random distribution, suggesting that the current sample of local CTAGNs is independent of host galaxy inclinations.

these wavelengths. The main results can be summarized as follows:

1. A broadband $(\approx 0.5-40 \mathrm{keV}) \mathrm{X}$-ray spectral analysis of the AGN using data from NuSTAR and Chandra observations reveals that our direct view toward the AGN is hindered by a CT column of obscuring material, with a column density of $N_{\mathrm{H}}(\mathrm{los}) \gtrsim 2.5 \times 10^{24} \mathrm{~cm}^{-2}$. The range of $2-10 \mathrm{keV}$ intrinsic luminosity measured between the best-fitting torus models is $L_{2-10}$, int $=(3.5-7.6) \times 10^{40} \mathrm{erg} \mathrm{s}^{-1}$, consistent with that predicted from our optical and MIR data. These results indicate that NGC 1448 is one of the lowest luminosity CTAGNs known. The identification of NGC 1448 as a faint CTAGN also demonstrates the depth to which we can find CTAGNs with NuSTAR as compared to Swift-BAT.

2. Optical spectroscopy performed using ESO NTT reclassifed the nuclear spectrum as a Seyfert galaxy based on BPT emission line diagnostics, thus identifying the AGN at optical wavelenghts for the first time.

3. MIR imaging conducted by Gemini/T-ReCS detected a compact nucleus, providing the first identification of the AGN at MIR continuum, further confirming the presence of an AGN in the galaxy.

4. Comparing the host galaxy inclination distribution of local CTAGNs with that expected from random galaxy orientations, we do not find any evidence that the incompleteness of the current CTAGN census is due to host galaxy inclination effects.

We thank the anonymous referee for useful comments which have helped to improve the paper. We acknowledge financial support from Majlis Amanah Rakyat (MARA) Malaysia (A.A.), the Science and Technology Facilities Council (STFC) grant ST/L00075X/1 (D.M.A.), ST/J003697/1 (P.G.), and ST/K501979/1 (G.B.L.). F.E.B. acknowledges support from CONICYT-Chile (Basal-CATA PFB-06/2007, FONDECYT Regular 1141218, "EMBIGGEN" Anillo ACT1101), and the 
Ministry of Economy, Development, and Tourism's Millennium Science Initiative through grant IC120009, awarded to The Millennium Institute of Astrophysics, MAS. P.B. would like to thank the STFC for funding. S.M.L.'s research was supported by an appointment to the NASA Postdoctoral Program at the NASA Goddard Space Flight Center, administered by the Universities Space Research Association under contract with NASA. M.K. acknowledges support from the Swiss National Science Foundation and Ambizione fellowship grant PZ00P2_154799/1. We acknowledge financial support from the CONICYT-Chile grants "EMBIGGEN" Anillo ACT1101 (C.R.), FONDECYT 1141218 (C.R.), BasalCATA PFB-06/2007 (C.R.) and from the China-CONICYT fund (C.R.).

NUSTAR is a project led by the California Institute of Technology (Caltech), managed by the Jet Propulsion Laboratory (JPL), and funded by the National Aeronautics and Space Administration (NASA). We thank the NuSTAR Operations, Software and Calibrations teams for support with these observations. This research has made use of the NuSTAR Data Analysis Software (NUSTARDAS) jointly developed by the ASI Science Data Center (ASDC, Italy) and the California Institute of Technology (USA). This research also made use of the data obtained through the High Energy Astrophysics Science Archive Research Center (HEASARC) Online Service, provided by the NASA/Goddard Space Flight Center, and the NASA/IPAC extragalactic Database (NED) operated by JPL, Caltech under contract with NASA.

Facilities: Chandra, Gemini:South, NTT, NuSTAR.

\section{References}

Akylas, A., Georgakakis, A., Georgantopoulos, I., Brightman, M., \& Nandra, K. 2012, A\&A, 546, A98

Annuar, A., Gandhi, P., Alexander, D. M., et al. 2015, ApJ, 815, 36

Antonucci, R. 1993, ARA\&A, 31, 473

Asmus, D., Gandhi, P., Hönig, S. F., Smette, A., \& Duschl, W. J. 2015, MNRAS, 454, 766

Asmus, D., Hönig, S. F., Gandhi, P., Smette, A., \& Duschl, W. J. 2014, MNRAS, 439, 1648

Baldwin, J. A., Phillips, M. M., \& Terlevich, R. 1981, PASP, 93, 5

Baloković, M., Comastri, A., Harrison, F. A., et al. 2014, ApJ, 794, 111

Bauer, F. E., Arévalo, P., Walton, D. J., et al. 2015, ApJ, 812, 116

Baumgartner, W. H., Tueller, J., Markwardt, C. B., et al. 2013, ApJS, 207, 19

Bell, E. F., McIntosh, D. H., Katz, N., \& Weinberg, M. D. 2003, ApJS, 149,289

Bennert, N., Jungwiert, B., Komossa, S., Haas, M., \& Chini, R. 2006, A\&A, 456, 953

Brandt, W. N., \& Alexander, D. M. 2015, A\&ARv, 23, 1

Brightman, M., Baloković, M., Stern, D., et al. 2015, ApJ, 805, 41

Brightman, M., \& Nandra, K. 2011, MNRAS, 413, 1206

Bruzual, G., \& Charlot, S. 2003, MNRAS, 344, 1000

Burlon, D., Ajello, M., Greiner, J., et al. 2011, ApJ, 728, 58

Cardelli, J. A., Clayton, G. C., \& Mathis, J. S. 1989, ApJ, 345, 245

Cash, W. 1979, ApJ, 228, 939

Corral, A., Della Ceca, R., Caccianiga, A., et al. 2011, A\&A, 530, A42

Davé, R. 2008, MNRAS, 385, 147

Draper, A. R., \& Ballantyne, D. R. 2010, ApJL, 715, L99

Elitzur, M., \& Shlosman, I. 2006, ApJL, 648, L101

Elvis, M. 2012, JPhCS, 372, 012032
Gandhi, P., Annuar, A., Lansbury, G. B., et al. 2016, MNRAS, submitted (arXiv:1605.08041)

Gandhi, P., Horst, H., Smette, A., et al. 2009, A\&A, 502, 457

Gandhi, P., Lansbury, G. B., Alexander, D. M., et al. 2014, ApJ, 792, 117

Gandhi, P., Yamada, S., Ricci, C., et al. 2015, MNRAS, 449, 1845

Gilfanov, M. 2004, MNRAS, 349, 146

Gilli, R., Comastri, A., \& Hasinger, G. 2007, A\&A, 463, 79

Goulding, A. D., \& Alexander, D. M. 2009, MNRAS, 398, 1165

Goulding, A. D., Alexander, D. M., Bauer, F. E., et al. 2012, ApJ, 755, 5

Goulding, A. D., Alexander, D. M., Lehmer, B. D., \& Mullaney, J. R. 2010 MNRAS, 406, 597

Goulding, A. D., Alexander, D. M., Mullaney, J. R., et al. 2011, MNRAS, 411,1231

Güver, T., \& Özel, F. 2009, MNRAS, 400, 2050

Harrison, F. A., Craig, W. W., Christensen, F. E., et al. 2013, ApJ, 770, 103

Hönig, S. F., \& Beckert, T. 2007, MNRAS, 380, 1172

Horst, H., Gandhi, P., Smette, A., \& Duschl, W. J. 2008, A\&A, 479, 389

Jarrett, T. H., Chester, T., Cutri, R., Schneider, S. E., \& Huchra, J. P. 2003, AJ, 125,525

Kalberla, P. M. W., Burton, W. B., Hartmann, D., et al. 2005, A\&A, 440, 775

Kauffmann, G., Heckman, T. M., Tremonti, C., et al. 2003, MNRAS, 346,1055

Kawamuro, T., Ueda, Y., Tazaki, F., Terashima, Y., \& Mushotzky, R. 2016, arXiv: 1604.07915

Kennicutt, R. C., Jr. 1998, ApJ, 498, 541

Kewley, L. J., Dopita, M. A., Sutherland, R. S., Heisler, C. A., \& Trevena, J. 2001, ApJ, 556, 121

Kewley, L. J., Groves, B., Kauffmann, G., \& Heckman, T. 2006, MNRAS, 372, 961

Koss, M., Mushotzky, R., Baumgartner, W., et al. 2013, ApJL, 765, L26

Koss, M. J., Assef, R., Balokovic, M., et al. 2016, ApJ, in press (arXiv:1604. 07825)

Lehmer, B. D., Xue, Y. Q., Brandt, W. N., et al. 2012, ApJ, 752, 46

Madsen, K. K., Harrison, F. A., Markwardt, C., et al. 2015, ApJS, 220, 8

Magdziarz, P., \& Zdziarski, A. A. 1995, MNRAS, 273, 837

Marconi, A., \& Hunt, L. K. 2003, ApJL, 589, L21

Mineo, S., Gilfanov, M., \& Sunyaev, R. 2012, MNRAS, 419, 2095

Monard, L. A. G., Kneip, R., Brimacombe, J., et al. 2014, CBET, 3977, 1

Mould, J. R., Huchra, J. P., Freedman, W. L., et al. 2000, ApJ, 529, 786

Murphy, K. D., \& Yaqoob, T. 2009, MNRAS, 397, 1549

Noguchi, K., Terashima, Y., Ishino, Y., et al. 2010, ApJ, 711, 144

Osterbrock, D. E. 1989, Astrophysics of Gaseous Nebulae and Active Galactic Nuclei (Mill Valley, CA: University Science Books)

Panessa, F., Bassani, L., Cappi, M., et al. 2006, A\&A, 455, 173

Prieto, M. A., Mezcua, M., Fernández-Ontiveros, J. A., \& Schartmann, M. 2014, MNRAS, 442, 2145

Puccetti, S., Comastri, A., Fiore, F., et al. 2014, ApJ, 793, 26

Ricci, C., Ueda, Y., Koss, M. J., et al. 2016, arXiv:1603.04852

Risaliti, G., Maiolino, R., \& Salvati, M. 1999, ApJ, 522, 157

Rivers, E., Baloković, M., Arévalo, P., et al. 2015, ApJ, 815, 55

Sanders, D. B., Mazzarella, J. M., Kim, D.-C., Surace, J. A., \& Soifer, B. T. 2003, AJ, 126, 1607

Smith, R. K., Brickhouse, N. S., Liedahl, D. A., \& Raymond, J. C. 2001, ApJL, 556, L91

Sollerman, J., Cox, N., Mattila, S., et al. 2005, A\&A, 429, 559

Telesco, C. M., Pina, R. K., Hanna, K. T., et al. 1998, Proc. SPIE, 3354, 534

Terashima, Y., Ptak, A., Fujimoto, R., et al. 1998, ApJ, 496, 210

Treister, E., Urry, C. M., \& Virani, S. 2009, ApJ, 696, 110

Tremonti, C. A., Heckman, T. M., Kauffmann, G., et al. 2004, ApJ, 613, 898

Ueda, Y., Akiyama, M., Hasinger, G., Miyaji, T., \& Watson, M. G. 2014, ApJ, 786, 104

Ueda, Y., Eguchi, S., Terashima, Y., et al. 2007, ApJL, 664, L79

Urry, C. M., \& Padovani, P. 1995, PASP, 107, 803

Vasudevan, R. V., Fabian, A. C., Gandhi, P., Winter, L. M., \& Mushotzky, R. F. 2010, MNRAS, 402, 1081

Veron-Cetty, M.-P., \& Veron, P. 1986, A\&AS, 66, 335

Wang, L., Baade, D., Höflich, P., et al. 2003, ApJ, 591, 1110 